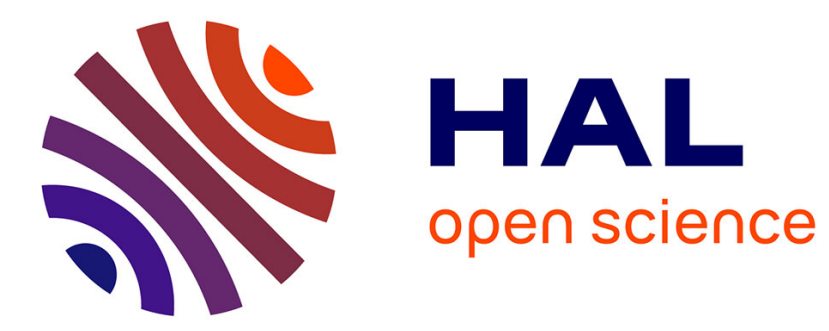

\title{
Poroelastic two-phase material modeling: theoretical formulation and embedded finite element method implementation
}

Nathan Benkemoun, Rachel Gelet, Emmanuel Roubin, Jean-Baptiste Colliat

\section{- To cite this version:}

Nathan Benkemoun, Rachel Gelet, Emmanuel Roubin, Jean-Baptiste Colliat. Poroelastic two-phase material modeling: theoretical formulation and embedded finite element method implementation. International Journal for Numerical and Analytical Methods in Geomechanics, 2015, 39 (12), pp.1255 - 1275. 10.1002/nag.2351 . hal-01380345

\section{HAL Id: hal-01380345 \\ https://hal.science/hal-01380345}

Submitted on 14 Oct 2016

HAL is a multi-disciplinary open access archive for the deposit and dissemination of scientific research documents, whether they are published or not. The documents may come from teaching and research institutions in France or abroad, or from public or private research centers.
L'archive ouverte pluridisciplinaire HAL, est destinée au dépôt et à la diffusion de documents scientifiques de niveau recherche, publiés ou non, émanant des établissements d'enseignement et de recherche français ou étrangers, des laboratoires publics ou privés. 


\title{
Poro-elastic two-phase material modeling: theoretical formulation and Embedded Finite Element Method implementation
}

\author{
Nathan Benkemoun* ${ }^{*}$ Rachel Gelet* ${ }^{*}$ Emmanuel Roubin ${ }^{\dagger}$, Jean-Baptiste Colliat ${ }^{\ddagger *}$ \\ * Institut de Recherche en Génie Civil et Mécanique (GeM), UMR CNRS 6183, \\ Université de Nantes, Ecole Centrale de Nantes \\ 58 rue Michel Ange, 44600 Saint-Nazaire, FRANCE \\ $\dagger$ LMT-Cachan, \\ Ecole Normale Supérieure de Cachan, Université Paris VI, CNRS, UniverSud Paris \\ 61 avenue du Président Wilson, 94235 Cachan Cedex, FRANCE \\ ¥ Laboratoire de Mécanique de Lille, \\ Université Sciences et Technologies Lille 1, CNRS, Ecole Centrale de Lille, Arts et Métiers ParisTech \\ Boulevard Paul Langevin, Cité Scientifique, 59655 Villeneuve d'Ascq Cedex, FRANCE
}

\begin{abstract}
This paper presents the formulation of finite element methods for the numerical modeling of a poro-elastic two-phase (aggregates/mixture phase) solid. The displacement and pressure fields are decomposed, following the EAS method, into a regular part and an enhanced part. This leads to discontinuous strain and pressure gradient fields allowing to capture the jump in mechanical and hydrical properties passing through the interface between the aggregates and the mixture phase. All these enhanced fields are treated in the context of the Embedded Finite Element Method through a local enhancement of the finite element interpolations as these jumps appear. The local character of these interpolations lead after a static condensation of the enhanced fields to a problem exhibiting the same structure as common poro-elastic finite element models, but incorporating now the mechanical and hydrical properties of a two-phase solid.
\end{abstract}

Keywords: E-FEM; EAS method; Weak Discontinuity; Poroelasticity; Darcy Law; Return Mapping; Operator Split; Static Condensation;

\footnotetext{
${ }^{*}$ Corresponding author.
} 


\section{Introduction}

\section{Introduction}

Interest has significantly increased over the last decades in the use of Enriched Finite Element Methods such as the Generalized Finite Element Method (G-FEM, [1], [2]), the 5 eXtended Finite Element Method (X-FEM, [3], [4], [5]) and the Embedded Finite Element Method (E-FEM, 6], [7], [8], [9]). These methods all seek to enrich the basis of the classical Finite Element shape functions by the means of specific enhancements in the displacement/strain fields for mechanical problems and/or in the pressure/pressure gradient, temperature/temperature gradient, concentration/concentration gradient fields for multiphysics problems for instance. It results in higher-performances finite elements capable of capturing physical discontinuity such as cracks, fractures, slip lines and material interfaces. In the literature, the aforementioned enhancements are in general sorted into two categories:

- strong enhancements - a jump within the displacement (and/or the pressure, the temperature, the concentration,...) fields is introduced by the means of a so-called strong discontinuity $([10])$;

- weak enhancements - a jump within the strain (and/or the pressure gradient, the temperature gradient, the concentration gradient,...) fields is introduced by the means of a so-called weak discontinuity ([11]).

On the one hand, due to the flexibility of their numerical implementation and the increase of their numerical efficiency, these tools allow the possibility to treat a broad variety of Civil Engineering problems in reasonable computational time. On the other hand, in many Civil Engineering applications, one has to deal with continuum mechanical problems which cannot be uniquely classified in the context of either "solid mechanics" or "fluid problems". In general, these applications fall into the categories of porous media (see [12], [13], [14] for instance). There is a broad variety of problems falling into this category including: geomaterials problems (soil, rock, concrete,...), dealing with the coupled solid deformation and pore-fluid flow behavior of liquid and/or gas into a porous solid skeleton. Thus, modeling these kinds of problems in the context of the aforementioned Enriched Finite Element 
Methods seems to be an appealing approach. Several applications concerning these coupled problems in porous media referenced in recent literature are worth noting. [15] develop a model of moisture transport in a fractured porous media, [16] present a two-scale (mesomacro) model of gas transport in a fractured concrete, [17] a coupled thermo-mechanical failure model in porous construction materials, [18] and [19] a fully coupled poro-plastic model in the presence of shear bands in a fully and partially saturated porous solid, [20] derive a two-scale (micro-macro) approach for fluid flow in a fractured porous media, [21] present a coupled model between water and chloride ion transport and stress driven failure and [22] simulate an hydraulically-driven fracture in a porous media. Finally, a classification of this literature is presented in Table1, It summarizes the models in relation with the performed enhancements in the fields of interest. We denote by $(\mathrm{S})$ a strong enhancement and by $(\mathrm{W})$ a weak one. We call $\boldsymbol{u}, p_{f}, q_{f}$ and $T$ the displacement field, the pressure field, the mass flux and the temperature, respectively .

\begin{tabular}{|c|c|c|c|c|c|}
\hline Models in the literature & \multicolumn{4}{|c|}{ Fields of interest } & \multirow{2}{*}{ Resulting type of enhancement } \\
\cline { 2 - 5 } & $\boldsymbol{u}$ & $p_{f}$ & $q_{f}$ & $T$ & \\
\hline$[15]$ & $(\mathrm{S})$ & $(\mathrm{S})^{*}$ or $(\mathrm{W})^{\dagger}$ & & & $\mathrm{S}^{*} \mathrm{~S}^{*}$ or S/W \\
\hline$[17]$ & $(\mathrm{S})$ & & & $(\mathrm{W})$ & $\mathrm{S} / \mathrm{W}$ \\
\hline$[18]$ and $[19]$ & $(\mathrm{S})$ & & $(\mathrm{S})$ & & $\mathrm{S} / \mathrm{S}$ \\
\hline$[20]$ & $(\mathrm{S})$ & $(\mathrm{W})$ & & & $\mathrm{S} / \mathrm{W}$ \\
\hline$[21]$ & $(\mathrm{S})$ & $(\mathrm{S})$ & & & $\mathrm{S} / \mathrm{S}$ \\
\hline$[22]$ & $(\mathrm{S})$ & $(\mathrm{W})$ & & & $\mathrm{S} / \mathrm{W}$ \\
\hline
\end{tabular}

Table 1: Type of enhancement performed in the literature (note that ${ }^{*}$ stands for the G-FEM using and ${ }^{\dagger}$ for the E-FEM). For instance, a strong enhancement in the mechanical problem (jump in the displacement field) and a weak enhancement in the fluid problem (jump in the pressure gradient field) yield an enhancement labeled as $\mathrm{S} / \mathrm{W}$

Regarding this literature, all these models, excepted ([16]), are used at the macroscopic scale, thus considering the material (cementitious one in general) as an homogeneous medium i.e. a one-phase material with mechanical and permeability or thermal properties. Indeed the 
explicit representation of heterogeneities (shapes, distribution, sizes and behavior) present at fine scales and having a significant role ([23], [24]) in most of the physical phenomenon (degradation, cracking, transport,...) observed at the macroscopic scale is not taken into account. In this paper, we propose to develop a model at a relevant scale for a reasonable cost in computation time but fine enough to take into account these heterogeneities: the development of a mesoscopic scale (eg. millimeter scale for concrete) model for a fully saturated porous media is proposed. At this scale for concrete, heterogeneities can be considered as aggregates inclusions embedded into a mixture phase and this mixture phase can be seen as a mortar matrix with pores fully saturated by water.

In order to explicitly take into account these aggregates (shapes and geometry), the authors turn to a method referred as non-adapted meshing process $([25])$. This consists in a unique homogeneous mesh whose nodes are placed independently from the morphology of the aggregates. A significant amount of computation time is saved at this stage. However this implies the presence of finite elements split into two parts each having different hydromechanical properties. Fig. 1 shows the whole domain $\Omega$ and some element split by $\Gamma_{d}$ into two parts $\left(\Omega_{e}^{\oplus}\right.$ and $\left.\Omega_{e}^{\ominus}\right)$. On $\Omega_{e}^{\oplus}$, we consider $E^{\oplus}, \nu^{\oplus}, \boldsymbol{\kappa}^{\oplus}$ and on $\Omega_{e}^{\ominus} E^{\ominus}, \nu^{\ominus}, \boldsymbol{\kappa}^{\ominus}$ which are respectively the Young modulus, the Poisson ratio and the permeability in each parts. In order to take into account this special kinematics in the classical linear elements, a weak enhancement is introduced both in the strain field and the pressure gradient field leading to a $\mathrm{W} / \mathrm{W}$ enhancement. This paper presents the methodology to introduce this $\mathrm{W} / \mathrm{W}$ enhancement both in the mechanical problem and the fluid problem in the context of the E-FEM, leading to a mesoscopic poro-elastic fully saturated two-phase model.

It is as follows : In Section 2, the mechanical problem is presented. It is based upon a Hu-Washizu formulation ([26]). After that the form of the enhanced fields (enhanced strain field and pressure gradient field) is detailed; their introduction in the strain and the pressure gradient fields is based upon the Enhanced Assumed Strain method ([27]). Finally the discretized form of this mechanical problem is obtained as a result of the Finite Element Method. In Section 3, the fluid problem to be solved is introduced. After a little work, the discretized form of this problem is obtained. In Section 4, the solving process to solve this 
coupled hydro-mechanical problem is presented : it lies on two levels of solving a local one for the enhanced unknowns ([28]) and a global one for the regular parts of the displacement and pressure fields.

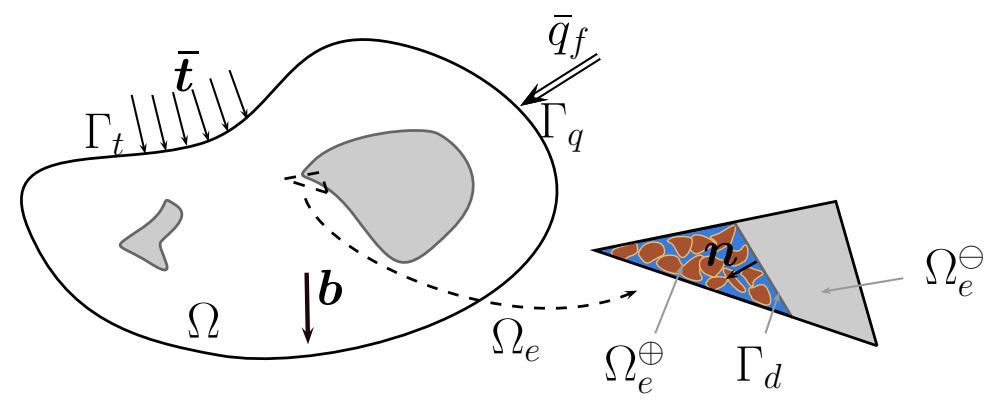

Figure 1: Illustration of the weak discontinuity in an element used in $\Omega_{e}: \Omega_{e}^{\ominus}$ represents the aggregate phase and $\Omega_{e}^{\oplus}$ the mixture phase (mortar matrix with pores fully saturated by water). The interface $\Gamma_{d}$ illustrates the position of the weak discontinuity

\section{Balance of linear momentum : modified $\mathrm{Hu}-$ Washizu formulation}

In this section, we first remind the mechanical problem to be solved. Then we show how to adapt it in the context of a porous media.

\subsection{Governing equations}

As mentioned in the introduction of this paper, some elements can be split into two parts, each part having different mechanical properties. This implies the introduction of a weak discontinuity $([25])$ in the kinematics of the strain field. It is achieved with the EAS method ([27]) considering both the actual and virtual strain fields decomposed into a regular part and an enhanced part. This gives for the actual strain field

$$
\boldsymbol{\varepsilon}=\underbrace{\boldsymbol{\nabla}^{s} \overline{\boldsymbol{u}}}_{\text {regular }}+\underbrace{\tilde{\boldsymbol{\varepsilon}}}_{\text {enhanced }}
$$

and for the virtual strain field

$$
\gamma=\underbrace{\nabla^{s} \overline{\boldsymbol{\eta}}}_{\text {regular }}+\underbrace{\tilde{\gamma}}_{\text {enhanced }},
$$


where $\boldsymbol{\nabla}^{s} \overline{\boldsymbol{u}}\left(\boldsymbol{\nabla}^{s} \overline{\boldsymbol{\eta}}\right)$ is the symmetric gradient of the actual (virtual) displacement field. As in $([27])$, we refer to $\tilde{\varepsilon}$ and $\tilde{\gamma}$ as the enhanced parts of the strain fields. The notation $(\tilde{\bullet}$ refers to weak discontinuity.

Introducing (11) and (2) in the Hu-Washizu formulation ([26]), one gets a modified $\mathrm{Hu}-$ Washizu formulation (see [29] for details) to be solved :

$$
\begin{aligned}
& \int_{\Omega} \nabla^{s} \overline{\boldsymbol{\eta}}: \check{\boldsymbol{\sigma}} d \Omega-\int_{\Omega} \overline{\boldsymbol{\eta}} \cdot \rho \boldsymbol{b} d \Omega-\int_{\Gamma_{t}} \overline{\boldsymbol{\eta}} \cdot \overline{\boldsymbol{t}} d \partial \Omega=0, \\
& \int_{\Omega_{e}} \tilde{\boldsymbol{\gamma}}: \check{\boldsymbol{\sigma}} d \Omega=0,\left(e=1,2, \ldots, n_{\text {elm }}^{\oplus, \ominus}\right)
\end{aligned}
$$

where $\check{\boldsymbol{\sigma}}$ is the total stress tensor, $\boldsymbol{b}$ the imposed volumetric body forces and $\overline{\boldsymbol{t}}$ the tractions applied at the boundary portion $\Gamma_{t}$. We note $n_{\text {elm }}^{\oplus, \ominus}$ the number of elements split into two parts $\Omega_{e}^{\oplus}$ and $\Omega_{e}^{\ominus}$.

Remark : Note that the first equation of the problem is global. It has to be solved for all the elements. On the contrary, the second one is local. It has to be solved only for elements split into two parts. This remark is very important for the resolution process described latter.

In the context of saturated porous media $([30])$, the total stress tensor $\check{\boldsymbol{\sigma}}$ is written such as

$$
\check{\boldsymbol{\sigma}}=\boldsymbol{\sigma}-p_{f} \mathbf{1}
$$

where $\boldsymbol{\sigma}$ is the effective stress tensor and $p_{f}$ the fluid pressure (positive in compression).

Combining (3a $)$, (3b) and (44) one obtains

$$
\begin{aligned}
& \int_{\Omega} \nabla^{s} \overline{\boldsymbol{\eta}}: \boldsymbol{\sigma} d \Omega-\int_{\Omega} p_{f} \nabla \cdot \overline{\boldsymbol{\eta}} d \Omega-\int_{\Omega} \overline{\boldsymbol{\eta}} \cdot \rho \boldsymbol{b} d \Omega-\int_{\Gamma_{t}} \overline{\boldsymbol{\eta}} \cdot \overline{\boldsymbol{t}} d \partial \Omega=0, \\
& \int_{\Omega_{e}} \tilde{\boldsymbol{\gamma}}:\left(\boldsymbol{\sigma}-p_{f} \mathbf{1}\right) d \Omega=0,\left(e=1,2, \ldots, n_{\text {elm }}^{\oplus, \ominus}\right) .
\end{aligned}
$$

Considering now the fluid problem, some elements can be split into two parts, each part having different properties of permeability. This implies, as for the mechanical problem, the introduction of a weak discontinuity in the kinematics of the pressure field. As for the mechanical problem, the symbol $(\boldsymbol{\bullet})$ refers to the regular part of the pressure and the symbol 
$(\tilde{\bullet})$ to the enhanced part (corresponding to the weak discontinuity) thus the actual pressure $p_{f}$ is such

$$
p_{f}=\underbrace{\bar{p}_{f}}_{\text {regular }}+\underbrace{\tilde{p}_{f}}_{\text {enhanced }} .
$$

Combining (5a), (5b), (6) and developing terms, one gets

$$
\begin{aligned}
& \int_{\Omega} \nabla^{s} \overline{\boldsymbol{\eta}}: \boldsymbol{\sigma} d \Omega-\int_{\Omega} \bar{p}_{f} \nabla \cdot \overline{\boldsymbol{\eta}} d \Omega-\int_{\Omega} \tilde{p}_{f} \nabla \cdot \overline{\boldsymbol{\eta}} d \Omega-\int_{\Omega} \overline{\boldsymbol{\eta}} \cdot \rho \boldsymbol{b} d \Omega-\int_{\Gamma_{t}} \overline{\boldsymbol{\eta}} \cdot \overline{\boldsymbol{t}} d \partial \Omega=0, \\
& \int_{\Omega_{e}} \tilde{\boldsymbol{\gamma}}: \boldsymbol{\sigma} d \Omega-\int_{\Omega_{e}} \tilde{\boldsymbol{\gamma}}:\left(\bar{p}_{f} \mathbf{1}\right) d \Omega-\int_{\Omega_{e}} \tilde{\boldsymbol{\gamma}}:\left(\tilde{p}_{f} \mathbf{1}\right) d \Omega=0,\left(e=1,2, \ldots, n_{\text {elm }}^{\oplus, \ominus}\right) .
\end{aligned}
$$

This represents the mechanical system to be solved. Nevertheless the form of the enhanced fields has still to be explicited. This is the focus of the next subsection.

\subsection{FE discretization of the fields}

In this subsection, we introduce the form of the enhanced fields present in the mechanical problem. Both continuous and discretized forms are introduced.

\subsubsection{Continuous form of the enhanced fields}

Following the idea developed in [31] and [29], the form of the enhanced displacement field is assumed to be

$$
\tilde{\boldsymbol{u}}=\Theta \boldsymbol{n} \cdot(\boldsymbol{x}-\boldsymbol{\xi})\left([\varepsilon]_{n} \boldsymbol{n}+[\varepsilon]_{m} \boldsymbol{m}+[\varepsilon]_{t} \boldsymbol{t}\right) \text { with } \Theta=\left\{\begin{array}{cc}
\Theta^{\oplus} & \forall \boldsymbol{x} \in \Omega_{e}^{\oplus} \\
\Theta^{\ominus} & \forall \boldsymbol{x} \in \Omega_{e}^{\ominus}
\end{array},\right.
$$

where $\boldsymbol{\xi}$ represents the position of the discontinuity $\Gamma_{d}, \Theta$ an unidentified shape function and $\left([\varepsilon]_{n},[\varepsilon]_{m},[\varepsilon]_{t}\right)$ three scalar values computed during the resolution process only for the elements split into two parts . The product $\boldsymbol{n} \cdot(\boldsymbol{x}-\boldsymbol{\xi})$ is called the distance function. Note that when $\boldsymbol{x}$ is equal to $\boldsymbol{\xi}$ (in other words when we are on the discontinuity $\Gamma_{d}$ ), $\tilde{\boldsymbol{u}}$ is equal to zero thus the displacement is continuous through the discontinuity.

Applying the symmetric gradient operator to $\tilde{\boldsymbol{u}}$, one gets

$$
\tilde{\boldsymbol{\varepsilon}}=\Theta\left([\varepsilon]_{n} \boldsymbol{n} \otimes \boldsymbol{n}+\frac{[\varepsilon]_{m}}{2}(\boldsymbol{n} \otimes \boldsymbol{m})^{s}+\frac{[\varepsilon]_{t}}{2}(\boldsymbol{n} \otimes \boldsymbol{t})^{s}\right) .
$$

Details are given in Appendix A. 
We now use the same reasoning to obtain the form of $\nabla \tilde{p}_{f}$ considering the fact that $\tilde{p}_{f}$ is a scalar field whereas $\tilde{\boldsymbol{u}}$ was a vectorial field.

We write $\tilde{p}_{f}$ under the following form

$$
\tilde{p}_{f}=\Theta \boldsymbol{n} \cdot(\boldsymbol{x}-\boldsymbol{\xi})\left(\tilde{p}_{f}^{h}\right)
$$

where $\boldsymbol{\xi}$ represents the position of $\Gamma_{d}$ and $\Theta$ an unidentified shape function. The product $\boldsymbol{n} \cdot(\boldsymbol{x}-\boldsymbol{\xi})$ is called the distance function. Note that when $\boldsymbol{x}$ is equal to $\boldsymbol{\xi}$ (in other words when we are on the discontinuity $\left.\Gamma_{d}\right), \tilde{p}_{f}$ is equal to zero thus the pressure is continuous through the discontinuity. $\tilde{p}_{f}^{h}$ is a scalar value computed during the resolution process only for the elements split into two parts.

Thus, following ([32]), the gradient of $\tilde{p}_{f}$ is given by

$$
\begin{aligned}
& \frac{\partial \tilde{p}_{f}}{\partial x_{1}}=\frac{\partial \tilde{p}_{f}}{\partial\left(n_{k}^{T} \cdot(x-\xi)_{k}\right)} \frac{\partial\left(n_{k}^{T} \cdot(x-\xi)_{k}\right)}{\partial x_{1}}=\Theta \tilde{p}_{f}^{h} n_{1}, \\
& \frac{\partial \tilde{p}_{f}}{\partial x_{2}}=\frac{\partial \tilde{p}_{f}}{\partial\left(n_{k}^{T} \cdot(x-\xi)_{k}\right)} \frac{\partial\left(n_{k}^{T} \cdot(x-\xi)_{k}\right)}{\partial x_{2}}=\Theta \tilde{p}_{f}^{h} n_{2}, \\
& \frac{\partial \tilde{p}_{f}}{\partial x_{3}}=\frac{\partial \tilde{p}_{f}}{\partial\left(n_{k}^{T} \cdot(x-\xi)_{k}\right)} \frac{\partial\left(n_{k}^{T} \cdot(x-\xi)_{k}\right)}{\partial x_{3}}=\Theta \tilde{p}_{f}^{h} n_{3} .
\end{aligned}
$$

Finally, one gets the form of $\nabla \tilde{p}_{f}$ such as

$$
\nabla \tilde{p}_{f}=\Theta \tilde{p}_{f}^{h}\left(\begin{array}{c}
n_{1} \\
n_{2} \\
n_{3}
\end{array}\right)=\Theta \tilde{p}_{f}^{h} \boldsymbol{n} .
$$

In the next subsection, we turn to the discretized form of $\tilde{\varepsilon}$ and $\nabla \tilde{p}_{f}$ suitable for a FE approach.

\subsubsection{Discretized form of the enhanced fields}

Following the idea presented in $([29]), \tilde{\varepsilon}$ is written under the form

$$
\tilde{\varepsilon}=G_{\mathrm{w}}^{u}[|\varepsilon|]
$$


where $\tilde{\varepsilon}$ is written under the Voigt notation and $[|\varepsilon|]$ is a vector containing $\left([\varepsilon]_{n},[\varepsilon]_{t},[\varepsilon]_{m}\right)$.

The form of $\boldsymbol{G}_{\mathrm{w}}^{u}$ is explicitly known

$$
\boldsymbol{G}_{\mathrm{w}}^{u}= \begin{cases}\boldsymbol{G}_{\mathrm{w}}^{u, \oplus}=\Theta^{\oplus} \boldsymbol{H}_{\mathrm{w}}^{u}=\frac{V^{\ominus}}{V} \boldsymbol{H}_{\mathrm{w}}^{u} & \text { in } \Omega_{e}^{\oplus} \\ \boldsymbol{G}_{\mathrm{w}}^{u, \ominus}=\Theta^{\ominus} \boldsymbol{H}_{\mathrm{w}}^{u}=-\frac{V^{\oplus}}{V} \boldsymbol{H}_{\mathrm{w}}^{u} & \text { in } \Omega_{e}^{\ominus}\end{cases}
$$

with

$$
\boldsymbol{H}_{\mathrm{w}}^{u}=\left[\begin{array}{ccc}
n_{1}^{2} & n_{1} m_{1} & n_{1} t_{1} \\
n_{2}^{2} & n_{2} m_{2} & n_{2} t_{2} \\
n_{3}^{2} & n_{3} m_{3} & n_{3} t_{3} \\
2 n_{1} n_{2} & n_{1} m_{2}+n_{2} m_{1} & n_{1} t_{2}+n_{2} t_{1} \\
2 n_{2} n_{3} & n_{2} m_{3}+n_{3} m_{2} & n_{2} t_{3}+n_{3} t_{2} \\
2 n_{1} n_{3} & n_{1} m_{3}+n_{3} m_{1} & n_{1} t_{3}+n_{3} t_{1}
\end{array}\right] .
$$

and $\Theta^{\oplus}=V^{\ominus} / V, \Theta^{\ominus}=-V^{\oplus} / V$ where $V, V^{\oplus}$ and $V^{\ominus}$ the volumes of $\Omega_{e}, \Omega_{e}^{\oplus}$ and $\Omega_{e}^{\ominus}$ respectively.

By analogy with the enhanced strain field, we write

$$
\nabla \tilde{p}_{f}=\boldsymbol{G}_{\mathrm{w}}^{p} \tilde{p}_{f}^{h}
$$

where $\nabla \tilde{p}_{f}$ is a column vector and $\tilde{p}_{f}^{h}$ a scalar value.

The form of $\boldsymbol{G}_{\mathrm{w}}^{p}$ is explicitly known

$$
\boldsymbol{G}_{\mathrm{w}}^{p}= \begin{cases}\boldsymbol{G}_{\mathrm{w}}^{p, \oplus}=\Theta^{\oplus} \boldsymbol{H}_{\mathrm{w}}^{p}=\frac{V^{\ominus}}{V} \boldsymbol{H}_{\mathrm{w}}^{p} & \text { in } \Omega_{e}^{\oplus} \\ \boldsymbol{G}_{\mathrm{w}}^{p, \ominus}=\Theta^{\ominus} \boldsymbol{H}_{\mathrm{w}}^{p}=-\frac{V^{\oplus}}{V} \boldsymbol{H}_{\mathrm{w}}^{p} & \text { in } \Omega_{e}^{\ominus}\end{cases}
$$

with

$$
\boldsymbol{H}_{\mathrm{w}}^{p}=\left[\begin{array}{c}
n_{1} \\
n_{2} \\
n_{3}
\end{array}\right] .
$$

Finally this gives for the enhanced pressure field

$$
\tilde{p}_{f}=M_{\mathrm{w}}^{p} \tilde{p}_{f}^{h},
$$

where $M_{\mathrm{w}}^{p}$ is a scalar function and $\tilde{p}_{f}^{h}$ a scalar value. 
The form of $M_{\mathrm{w}}^{p}$ is explicitly known

$$
M_{\mathrm{w}}^{p}=\left\{\begin{array}{ll}
M_{\mathrm{w}}^{p, \oplus}=\Theta^{\oplus} \boldsymbol{n} \cdot\left(\boldsymbol{x}^{\oplus}-\boldsymbol{\xi}\right)=\frac{V^{\ominus}}{V} \boldsymbol{n} \cdot\left(\boldsymbol{x}^{\oplus}-\boldsymbol{\xi}\right) & \text { in } \Omega_{e}^{\oplus} \\
M_{\mathrm{w}}^{p, \ominus}=\Theta^{\ominus} \boldsymbol{n} \cdot\left(\boldsymbol{x}^{\ominus}-\boldsymbol{\xi}\right)=-\frac{V^{\oplus}}{V} \boldsymbol{n} \cdot\left(\boldsymbol{x}^{\ominus}-\boldsymbol{\xi}\right) & \text { in } \Omega_{e}^{\ominus}
\end{array} .\right.
$$

Having the discretized form of the enhanced fields in hand, we now turn to the discretized form of the regular fields.

\subsubsection{Discretized form of the regular fields}

For the gradient of the regulart part of the virtual strain $\nabla^{s} \overline{\boldsymbol{\eta}}$, we have

$$
\nabla^{s} \overline{\boldsymbol{\eta}}=\mathcal{B}^{u} \overline{\boldsymbol{\eta}}^{h}
$$

where $\mathcal{B}^{u}$ is the classical interpolation matrix (derivatives) for a tetrahedron elements and $\overline{\boldsymbol{\eta}}^{h}$ is a column vector containing the virtual nodal displacement unknowns. Note that $\nabla^{s} \overline{\boldsymbol{\eta}}$ is again under the Voigt convention.

For the divergence of the regulart part of the virtual displacement $\nabla \cdot \overline{\boldsymbol{\eta}}$, we get

$$
\nabla \cdot \overline{\boldsymbol{\eta}}=\left[\begin{array}{llllll}
1 & 1 & 1 & 0 & 0 & 0
\end{array}\right] \nabla^{s} \overline{\boldsymbol{\eta}}=\boldsymbol{m} \nabla^{s} \overline{\boldsymbol{\eta}}=\boldsymbol{m} \mathcal{B}^{u} \overline{\boldsymbol{\eta}}^{h}
$$

For the regular part of the actual pressure field $\tilde{p}_{f}$, we have

$$
\bar{p}_{f}=\boldsymbol{N}^{p} \overline{\boldsymbol{p}}_{\boldsymbol{f}}^{h},
$$

where $\boldsymbol{N}^{p}$ is a row vector containing the shape functions for the regular part of the actual pressure field and $\overline{\boldsymbol{p}}_{\boldsymbol{f}}{ }^{h}$ is a column vector containing the actual nodal pressure unknowns.

Finally we obtain

$$
\begin{gathered}
\nabla^{s} \overline{\boldsymbol{\eta}}=\mathcal{B}^{u} \overline{\boldsymbol{\eta}}^{h}+\boldsymbol{G}_{\mathrm{w}}^{u}[|\boldsymbol{\gamma}|], \\
p_{f}=\boldsymbol{N}^{p} \overline{\boldsymbol{p}}_{f}{ }^{h}+M_{\mathrm{w}}^{p} \tilde{p}_{f}^{h}, \\
\nabla p_{f}=\boldsymbol{B}^{p} \overline{\boldsymbol{p}}_{\boldsymbol{f}}{ }^{h}+\boldsymbol{G}_{\mathrm{w}}^{p} \tilde{p}_{f}^{h},
\end{gathered}
$$


where $\boldsymbol{B}^{p}$ is a matrix containing the derivatives of the shape functions $\boldsymbol{N}^{p}$.

Note that in the expression of $\nabla^{s} \overline{\boldsymbol{\eta}}$, the enhanced part $\tilde{\boldsymbol{\gamma}}$ has the same form as $\tilde{\boldsymbol{\varepsilon}}$ where $[|\gamma|]=\left([\gamma]_{n},[\gamma]_{m},[\gamma]_{t}\right)$

Having the expression of $\nabla^{s} \overline{\boldsymbol{\eta}}, p_{f}$ and $\nabla p_{f}$ in a suitable form for a FE approach, we can now introduce them into the mechanical problem (7a) and (7b) and obtain the discretized mechanical problem to be solved.

\subsection{FE discretization of (7a) and (7b)}

Combining (24), (26), (27) and (7a) and considering the behaviour law for the effective stress such as

$$
\boldsymbol{\sigma}\left(\overline{\boldsymbol{u}}^{h},[|\varepsilon|]\right)= \begin{cases}\boldsymbol{\sigma}^{\oplus}=\boldsymbol{C}^{\oplus} \boldsymbol{\varepsilon}=\boldsymbol{C}^{\oplus}\left(\mathcal{B}^{u} \overline{\boldsymbol{u}}^{h}+\boldsymbol{G}_{\mathrm{w}}^{u, \oplus}[|\varepsilon|]\right) & \text { if } \boldsymbol{x} \in \Omega_{e}^{\oplus} \\ \boldsymbol{\sigma}^{\ominus}=\boldsymbol{C}^{\ominus} \boldsymbol{\varepsilon}=\boldsymbol{C}^{\ominus}\left(\mathcal{B}^{u} \overline{\boldsymbol{u}}^{h}+\boldsymbol{G}_{\mathrm{w}}^{u, \ominus}[|\varepsilon|]\right) & \text { if } \boldsymbol{x} \in \Omega_{e}^{\ominus}\end{cases}
$$

yield

$$
\begin{aligned}
& {\left[\int_{\Omega^{\oplus}} \mathcal{B}^{u, T} \boldsymbol{C}^{\oplus} \mathcal{B}^{u} d \Omega+\int_{\Omega^{\ominus}} \mathcal{B}^{u, T} \boldsymbol{C}^{\ominus} \mathcal{B}^{u} d \Omega\right] \overline{\boldsymbol{u}}^{h}+\left[\int_{\Omega^{\oplus}} \mathcal{B}^{u, T} \boldsymbol{C}^{\oplus} \boldsymbol{G}_{\mathrm{w}}^{u, \oplus} d \Omega\right.} \\
& \left.+\int_{\Omega^{\ominus}} \mathcal{B}^{u, T} \boldsymbol{C}^{\ominus} \boldsymbol{G}_{\mathrm{w}}^{u, \ominus} d \Omega\right][|\varepsilon|]-\left[\int_{\Omega} \mathcal{B}^{u, T} \boldsymbol{m}^{T} \boldsymbol{N}^{p} d \Omega\right] \overline{\boldsymbol{p}}_{\boldsymbol{f}}^{h}-\left[\int_{\Omega^{\oplus}} \mathcal{B}^{u, T} \boldsymbol{m}^{T} M_{\mathrm{w}}^{p, \oplus} d \Omega\right. \\
& \left.+\int_{\Omega^{\ominus}} \mathcal{B}^{u, T} \boldsymbol{m}^{T} M_{\mathrm{w}}^{p, \ominus} d \Omega\right] \tilde{p}_{f}^{h}-\int_{\Omega} \boldsymbol{N}^{u, T} \rho \boldsymbol{b} d \Omega-\int_{\Gamma_{t}} \boldsymbol{N}^{u, T} \overline{\boldsymbol{t}} d \partial \Omega=\mathbf{0} .
\end{aligned}
$$

where $\boldsymbol{C}^{\oplus}$ and $\boldsymbol{C}^{\ominus}$ are the elastic tensors for the material in $\Omega^{\oplus}$ and $\Omega^{\ominus}$.

Under matricial format, one gets

$$
\boldsymbol{K}_{\boldsymbol{u u}} \overline{\boldsymbol{u}}^{h}+\boldsymbol{K}_{\boldsymbol{u} \mathrm{w}}[|\varepsilon|]-\boldsymbol{K}_{\boldsymbol{u} \overline{\boldsymbol{p}}} \overline{\boldsymbol{p}}_{\boldsymbol{f}}^{h}-\boldsymbol{K}_{\boldsymbol{u} \tilde{p}} \tilde{p}_{f}^{h}=\boldsymbol{f}_{\boldsymbol{e x \boldsymbol { t }}},
$$

where

$$
\begin{gathered}
\boldsymbol{K}_{\boldsymbol{u u}}=\int_{\Omega^{\oplus}} \mathcal{B}^{u, T} \boldsymbol{C}^{\oplus} \mathcal{B}^{u} d \Omega+\int_{\Omega^{\ominus}} \mathcal{B}^{u, T} \boldsymbol{C}^{\ominus} \mathcal{B}^{u} d \Omega \\
\boldsymbol{K}_{\boldsymbol{u} \mathrm{w}}=\int_{\Omega^{\oplus}} \mathcal{B}^{u, T} \boldsymbol{C}^{\oplus} \boldsymbol{G}_{\mathrm{w}}^{u, \oplus} d \Omega+\int_{\Omega^{\ominus}} \mathcal{B}^{u, T} \boldsymbol{C}^{\ominus} \boldsymbol{G}_{\mathrm{w}}^{u, \ominus} d \Omega, \\
\boldsymbol{K}_{\boldsymbol{u} \overline{\boldsymbol{p}}}=\int_{\Omega} \mathcal{B}^{u, T} \boldsymbol{m}^{T} \boldsymbol{N}^{p} d \Omega
\end{gathered}
$$




$$
\begin{gathered}
\boldsymbol{K}_{\boldsymbol{u} \tilde{p}}=\int_{\Omega^{\oplus}} \mathcal{B}^{u, T} \boldsymbol{m}^{T} M_{\mathrm{w}}^{p, \oplus} d \Omega+\int_{\Omega^{\ominus}} \mathcal{B}^{u, T} \boldsymbol{m}^{T} M_{\mathrm{w}}^{p, \ominus} d \Omega, \\
\boldsymbol{f}_{\text {ext }}=\int_{\Omega} \boldsymbol{N}^{u, T} \rho \boldsymbol{b} d \Omega+\int_{\Gamma_{t}} \boldsymbol{N}^{u, T} \overline{\boldsymbol{t}} d \partial \Omega .
\end{gathered}
$$

Combining (24), (26)

$$
\begin{aligned}
& {\left[\int_{\Omega_{e}^{\oplus}} \boldsymbol{G}_{\mathrm{w}}^{u, \oplus, T} \boldsymbol{C}^{\oplus} \mathcal{B}^{u} d \Omega+\int_{\Omega_{e}^{\ominus}} \boldsymbol{G}_{\mathrm{w}}^{u, \ominus, T} \boldsymbol{C}^{\ominus} \mathcal{B}^{u} d \Omega\right] \overline{\boldsymbol{u}}^{h}+\left[\int_{\Omega_{e}^{\oplus}} \boldsymbol{G}_{\mathrm{w}}^{u, \oplus, T} \boldsymbol{C}^{\oplus} \boldsymbol{G}_{\mathrm{w}}^{u, \oplus} d \Omega\right.} \\
& \left.+\int_{\Omega_{e}^{\ominus}} \boldsymbol{G}_{\mathrm{w}}^{u, \ominus, T} \boldsymbol{C}^{\ominus} \boldsymbol{G}_{\mathrm{w}}^{u, \ominus} d \Omega\right][|\boldsymbol{\varepsilon}|]-\left[\int_{\Omega_{e}^{\oplus}} \boldsymbol{G}_{\mathrm{w}}^{u, \oplus, T} \boldsymbol{m}^{T} \boldsymbol{N}^{p} d \Omega+\int_{\Omega_{e}^{\ominus}} \boldsymbol{G}_{\mathrm{w}}^{u, \ominus, T} \boldsymbol{m}^{T} \boldsymbol{N}^{p} d \Omega\right] \overline{\boldsymbol{p}}_{\boldsymbol{f}}^{h} \\
& -\left[\int_{\Omega_{e}^{\oplus}} \boldsymbol{G}_{\mathrm{w}}^{u, \oplus, T} \boldsymbol{m}^{T} M_{\mathrm{w}}^{p, \oplus} d \Omega+\int_{\Omega_{e}^{\ominus}} \boldsymbol{G}_{\mathrm{w}}^{u, \ominus, T} \boldsymbol{m}^{T} M_{\mathrm{w}}^{p, \ominus} d \Omega\right] \tilde{p}_{f}^{h}=\mathbf{0} .
\end{aligned}
$$

Under a matricial format, one gets

$$
\boldsymbol{K}_{\boldsymbol{u}}^{T}{ }^{T} \overline{\boldsymbol{u}}^{h}+\boldsymbol{K}_{\mathrm{ww}}[|\varepsilon|]-\boldsymbol{K}_{\mathrm{w} \overline{\boldsymbol{p}}} \overline{\boldsymbol{p}}_{\boldsymbol{f}}^{h}-\boldsymbol{K}_{\mathrm{w} \tilde{p}} \tilde{p}_{f}^{h}=\mathbf{0}
$$

where

$$
\begin{gathered}
\boldsymbol{K}_{\mathrm{ww}}=\int_{\Omega_{e}^{\oplus}} \boldsymbol{G}_{\mathrm{w}}^{u, \oplus, T} \boldsymbol{C}^{\oplus} \boldsymbol{G}_{\mathrm{w}}^{u, \oplus} d \Omega+\int_{\Omega_{e}^{\ominus}} \boldsymbol{G}_{\mathrm{w}}^{u, \ominus, T} \boldsymbol{C}^{\ominus} \boldsymbol{G}_{\mathrm{w}}^{u, \ominus} d \Omega, \\
\boldsymbol{K}_{\mathrm{w} \overline{\boldsymbol{p}}}=\int_{\Omega_{e}^{\oplus}} \boldsymbol{G}_{\mathrm{w}}^{u, \oplus, T} \boldsymbol{m}^{T} \boldsymbol{N}^{p} d \Omega+\int_{\Omega_{e}^{\ominus}} \boldsymbol{G}_{\mathrm{w}}^{u, \ominus, T} \boldsymbol{m}^{T} \boldsymbol{N}^{p} d \Omega, \\
\boldsymbol{K}_{\mathrm{w} \tilde{p}}=\int_{\Omega_{e}^{\oplus}} \boldsymbol{G}_{\mathrm{w}}^{u, \oplus, T} \boldsymbol{m}^{T} M_{\mathrm{w}}^{p, \oplus} d \Omega+\int_{\Omega_{e}^{\ominus}} \boldsymbol{G}_{\mathrm{w}}^{u, \ominus, T} \boldsymbol{m}^{T} M_{\mathrm{w}}^{p, \ominus} d \Omega .
\end{gathered}
$$

\section{Balance of mass equation : two-phase mixture formulation}

\subsection{Governing equations}

In the present work, the model is formulated in a Biot thermodynamic framework ([33], 
the development made in $([30])$, the weak form of the mass balance equation for the twophase mixture (mortar matrix fully saturated by water) can be written

$$
\int_{\Omega} \psi \frac{\dot{p_{f}}}{Q} d \Omega+\int_{\Omega} \psi \nabla \cdot \dot{\boldsymbol{u}}_{s} d \Omega+\int_{\Gamma} \psi \boldsymbol{v} \cdot \boldsymbol{n} d \partial \Omega+\frac{1}{g \rho_{w}} \int_{\Omega} \nabla \psi \cdot \boldsymbol{\kappa} \nabla p_{f} d \Omega-\frac{1}{g} \int_{\Omega} \nabla \psi \cdot \boldsymbol{\kappa} \boldsymbol{b} d \Omega=0
$$

where $\psi$ is the virtual pressure field, vanishing on $\Gamma_{p}$ where the pressure $p_{f}$ is imposed, $Q$ the ratio between the porosity $n$ and the pore water compressibility $K_{w}$ and $\boldsymbol{u}_{s}$ the solid-skeleton velocity. We note the part of the boundary $\Gamma_{q}$ where the external normal flow $\boldsymbol{v} \cdot \boldsymbol{n}_{q}$ for the outward unit normal $\boldsymbol{n}_{q}$ to $d \partial \Omega$, is $-\bar{q}_{f}$. Finally we consider $\Gamma_{d}$ the weak discontinuity surface splitting a given element into two-subdomains $\Omega^{\oplus}$ and $\Omega^{\ominus}$ where in terms of fluxes we have $\boldsymbol{v} \cdot \boldsymbol{n}_{d}^{\oplus}=-\bar{q}_{f}^{\oplus}$ and $\boldsymbol{v} \cdot \boldsymbol{n}_{d}^{\ominus}=-\bar{q}_{f}^{\ominus}$. Note that $\boldsymbol{n}_{d}^{\oplus}$ is pointing toward $\Omega^{\oplus}$ and $\boldsymbol{n}_{d}^{\ominus}$ toward $\Omega^{\ominus}$.

With these considerations in hands, (42) can be rewritten

$$
\begin{aligned}
& \int_{\Omega} \psi \frac{p_{f}}{Q} d \Omega+\int_{\Omega} \psi \nabla \cdot \dot{\boldsymbol{u}}_{s} d \Omega+\int_{\Gamma_{q}} \psi \boldsymbol{v} \cdot \boldsymbol{n}_{q} d \partial \Omega+\int_{\Gamma_{d}} \psi \boldsymbol{v} \cdot \boldsymbol{n}_{d} d \partial \Omega \\
& +\frac{1}{g \rho_{w}} \int_{\Omega} \nabla \psi \cdot \boldsymbol{\kappa} \nabla p_{f} d \Omega-\frac{1}{g} \int_{\Omega} \nabla \psi \cdot \boldsymbol{\kappa} \boldsymbol{b} d \Omega=0 .
\end{aligned}
$$

The part on $\Gamma_{d}$ can be decomposed into

$$
\begin{aligned}
\int_{\Gamma_{d}} \psi \boldsymbol{v} \cdot \boldsymbol{n}_{d} d \partial \Omega & =\int_{\Gamma_{d}^{\oplus}} \psi \boldsymbol{v} \cdot \boldsymbol{n}_{d}^{\oplus} d \partial \Omega+\int_{\Gamma_{d}^{\ominus}} \psi \boldsymbol{v} \cdot \boldsymbol{n}_{d}^{\ominus} d \partial \Omega \\
& =-\int_{\Gamma_{d}^{\oplus}} \psi \bar{q}_{f}^{\oplus} d \partial \Omega-\int_{\Gamma_{d}^{\ominus}} \psi \bar{q}_{f}^{\ominus} d \partial \Omega .
\end{aligned}
$$

In the case of a weak discontinuity, we consider that no flux is lost passing through the discontinuity $\Gamma_{d}$ thus $\bar{q}_{f}^{\oplus}+\bar{q}_{f}^{\ominus}=0$. This implies that

$$
-\int_{\Gamma_{d}^{\oplus}} \psi \bar{q}_{f}^{\oplus} d \partial \Omega-\int_{\Gamma_{d}^{\ominus}} \psi \bar{q}_{f}^{\ominus} d \partial \Omega=0,
$$

and so

$$
\int_{\Gamma_{d}} \psi \boldsymbol{v} \cdot \boldsymbol{n}_{d} d \partial \Omega=0
$$


Finally combining (43) and (46), one has

$$
\int_{\Omega} \psi \frac{\dot{p_{f}}}{Q} d \Omega+\int_{\Omega} \psi \nabla \cdot \dot{\boldsymbol{i}}_{s} d \Omega-\int_{\Gamma_{q}} \psi \bar{q}_{f} d \partial \Omega+\frac{1}{g \rho_{w}} \int_{\Omega} \nabla \psi \cdot \boldsymbol{\kappa} \nabla p_{f} d \Omega-\frac{1}{g} \int_{\Omega} \nabla \psi \cdot \boldsymbol{\kappa} \boldsymbol{b} d \Omega=0 .
$$

Following the form of $p_{f}$, the virtual pressure field $\psi$ is decomposed such as

$$
\psi=\underbrace{\bar{\psi}}_{\text {regular }}+\underbrace{\tilde{\psi}}_{\text {enhanced }} .
$$

The shape functions are the same as for the actual pressure field thus

$$
\begin{gathered}
\psi=\boldsymbol{N}^{p} \overline{\boldsymbol{\psi}}^{h}+M_{\mathrm{w}}^{p} \tilde{\psi}^{h}, \\
\nabla \psi=\boldsymbol{B}^{p} \overline{\boldsymbol{\psi}}^{h}+\boldsymbol{G}_{\mathrm{w}}^{p} \tilde{\psi}^{h},
\end{gathered}
$$

where $\boldsymbol{B}^{p}$ is a matrix containing the derivatives of the shape functions $\boldsymbol{N}^{p}$.

Having in hands the discretized form of the fields present in (47), we now turn to its discretization.

\subsection{FE discretization of (47)}

To carry out the time integration in (47), a backward Euler method is adopted:

$$
\left(\frac{d(\cdot)}{d t}\right)_{n+1}=\frac{(\cdot)_{n+1}-(\cdot)_{n}}{\Delta t}=\frac{\Delta(\cdot)}{\Delta t}
$$

where $\Delta t$ is the time increment, while $(\cdot)_{n+1}$ and $(\cdot)_{n}$ denote the unknowns at time step $n+1$ and $n$, respectively.

Combining (47) with (49), (150), (27) and (28), considering the fact that $\nabla \cdot \boldsymbol{u}_{s}=\boldsymbol{m} \dot{\varepsilon}$ and using (51) for the time derivatives, one gets $\forall \bar{\psi}^{h, T}$

$$
\begin{aligned}
& {\left[\frac{1}{Q} \int_{\Omega} \frac{1}{\Delta t} \boldsymbol{N}^{p, T} \boldsymbol{N}^{p} d \Omega\right] \Delta \overline{\boldsymbol{p}}_{\boldsymbol{f}}^{h}+\left[\frac{1}{g \rho_{w}} \int_{\Omega^{\oplus}} \boldsymbol{B}^{p, T} \cdot \boldsymbol{\kappa}^{\oplus} \boldsymbol{B}^{p} d \Omega+\frac{1}{g \rho_{w}} \int_{\Omega^{\ominus}} \boldsymbol{B}^{p, T} \cdot \boldsymbol{\kappa}^{\ominus} \boldsymbol{B}^{p} d \Omega\right] \overline{\boldsymbol{p}}_{\boldsymbol{f}}^{h}} \\
& +\left[\frac{1}{Q} \int_{\Omega^{\oplus}} \frac{1}{\Delta t} \boldsymbol{N}^{p, T} M_{\mathrm{w}}^{p, \oplus} d \Omega+\frac{1}{Q} \int_{\Omega^{\ominus}} \frac{1}{\Delta t} \boldsymbol{N}^{p, T} M_{\mathrm{w}}^{p, \ominus} d \Omega\right] \Delta \tilde{p}_{f}^{h}+\left[\frac{1}{g \rho_{w}} \int_{\Omega^{\oplus}} \boldsymbol{B}^{p, T} \cdot \boldsymbol{\kappa}^{\oplus} \boldsymbol{G}_{\mathrm{w}}^{p, \oplus} d \Omega\right. \\
& \left.+\frac{1}{g \rho_{w}} \int_{\Omega^{\ominus}} \boldsymbol{B}^{p, T} \cdot \boldsymbol{\kappa}^{\ominus} \boldsymbol{G}_{\mathrm{w}}^{p, \ominus} d \Omega\right] \tilde{p}_{f}^{h}+\left[\int_{\Omega} \frac{1}{\Delta t} \boldsymbol{N}^{p, T} \boldsymbol{m} \mathcal{B}^{u} d \Omega\right] \Delta \overline{\boldsymbol{u}}^{h}+\left[\int_{\Omega^{\oplus}} \frac{1}{\Delta t} \boldsymbol{N}^{p, T} \boldsymbol{m} \boldsymbol{G}_{\mathrm{w}}^{u, \oplus} d \Omega\right. \\
& \left.+\int_{\Omega^{\ominus}} \frac{1}{\Delta t} \boldsymbol{N}^{p, T} \boldsymbol{m} \boldsymbol{G}_{\mathrm{w}}^{u, \ominus} d \Omega\right] \Delta[|\boldsymbol{\varepsilon}|]-\int_{\Gamma_{q}} \boldsymbol{N}^{p, T} \bar{q}_{f} d \partial \Omega-\frac{1}{g} \int_{\Omega} \boldsymbol{B}^{p, T} \cdot \boldsymbol{\kappa} \boldsymbol{b} d \Omega=\mathbf{0},
\end{aligned}
$$


and $\forall \tilde{\psi}^{h, T}$

$$
\begin{aligned}
& {\left[\frac{1}{Q} \int_{\Omega_{e}^{\oplus}} \frac{1}{\Delta t} M_{\mathrm{w}}^{p, \oplus} \boldsymbol{N}^{p} d \Omega+\frac{1}{Q} \int_{\Omega_{e}^{\ominus}} \frac{1}{\Delta t} M_{\mathrm{w}}^{p, \ominus} \boldsymbol{N}^{p} d \Omega\right] \Delta \overline{\boldsymbol{p}}_{f}^{h}+\left[\frac{1}{g \rho_{w}} \int_{\Omega_{e}^{\oplus}} \boldsymbol{G}_{\mathrm{w}}^{p, \oplus, T} \cdot \boldsymbol{\kappa}^{\oplus} \boldsymbol{B}^{p} d \Omega\right.} \\
& \left.+\frac{1}{g \rho_{w}} \int_{\Omega_{e}^{\ominus}} \boldsymbol{G}_{\mathrm{w}}^{p, \ominus, T} \cdot \boldsymbol{\kappa}^{\ominus} \boldsymbol{B}^{p} d \Omega\right] \overline{\boldsymbol{p}}_{\boldsymbol{f}}^{h}+\left[\frac{1}{Q} \int_{\Omega_{e}^{\oplus}} \frac{1}{\Delta t} M_{\mathrm{w}}^{p, \oplus, T} M_{\mathrm{w}}^{p, \oplus} d \Omega+\frac{1}{Q} \int_{\Omega_{e}^{\ominus}} \frac{1}{\Delta t} M_{\mathrm{w}}^{p, \ominus, T} M_{\mathrm{w}}^{p, \ominus} d \Omega\right] \Delta \tilde{p}_{f}^{h} \\
& +\left[\frac{1}{g \rho_{w}} \int_{\Omega_{e}^{\oplus}} \boldsymbol{G}_{\mathrm{w}}^{p, \oplus, T} \cdot \boldsymbol{\kappa}^{\oplus} \boldsymbol{G}_{\mathrm{w}}^{p, \oplus} d \Omega+\frac{1}{g \rho_{w}} \int_{\Omega_{e}^{\ominus}} \boldsymbol{G}_{\mathrm{w}}^{p, \ominus, T} \cdot \boldsymbol{\kappa}^{\ominus} \boldsymbol{G}_{\mathrm{w}}^{p, \ominus} d \Omega\right] \tilde{p}_{f}^{h}+\left[\int_{\Omega_{e}^{\oplus}} \frac{1}{\Delta t} M_{\mathrm{w}}^{p, \oplus, T} \boldsymbol{m} \mathcal{B}^{u} d \Omega\right. \\
& \left.+\int_{\Omega_{e}^{\ominus}} \frac{1}{\Delta t} M_{\mathrm{w}}^{p, \ominus, T} \boldsymbol{m} \mathcal{B}^{u} d \Omega\right] \Delta \overline{\boldsymbol{u}}^{h}+\left[\int_{\Omega_{e}^{\oplus}} \frac{1}{\Delta t} M_{\mathrm{w}}^{p, \oplus, T} \boldsymbol{m} \boldsymbol{G}_{\mathrm{w}}^{u, \oplus} d \Omega+\int_{\Omega_{e}^{\ominus}} \frac{1}{\Delta t} M_{\mathrm{w}}^{p, \ominus, T} \boldsymbol{m} \boldsymbol{G}_{\mathrm{w}}^{u, \ominus} d \Omega\right] \Delta[|\boldsymbol{\varepsilon}|] \\
& -\int_{\Gamma_{q}} M_{\mathrm{w}}^{p, T} \bar{q}_{f} d \partial \Omega-\frac{1}{g} \int_{\Omega_{e}} \boldsymbol{G}_{\mathrm{w}}^{p, T} \cdot \boldsymbol{\kappa} \boldsymbol{b} d \Omega=0,\left(e=1,2, \ldots, n_{e l m}^{\oplus, \ominus}\right) .
\end{aligned}
$$

Under a matricial format, (52) and (531) are rewritten such as

$$
\begin{aligned}
& \frac{1}{\Delta t} \boldsymbol{K}_{\boldsymbol{u} \overline{\boldsymbol{p}}}^{T} \Delta \overline{\boldsymbol{u}}^{h}+\frac{1}{\Delta t} \boldsymbol{K}_{\mathrm{w} \overline{\boldsymbol{p}}}^{T} \Delta[|\boldsymbol{\varepsilon}|]+\frac{1}{\Delta t} \mathcal{M} \Delta \overline{\boldsymbol{p}}_{f}{ }^{h}+\mathcal{H} \overline{\boldsymbol{p}}_{\boldsymbol{f}}{ }^{h}+\frac{1}{\Delta t} \mathcal{L} \Delta \tilde{p}_{f}^{h}+\mathcal{G} \tilde{p}_{f}^{h}=\boldsymbol{f}_{\mathbf{l}_{\text {ext }}}, \\
& \frac{1}{\Delta t} \boldsymbol{K}_{\boldsymbol{u} \tilde{p}}^{T} \Delta \overline{\boldsymbol{u}}^{h}+\frac{1}{\Delta t} \boldsymbol{K}_{\mathrm{w} \tilde{p}}^{T} \Delta[|\boldsymbol{\varepsilon}|]+\frac{1}{\Delta t} \mathcal{L}^{T} \Delta \overline{\boldsymbol{p}}_{f}{ }^{h}+\mathcal{G}^{T} \overline{\boldsymbol{p}}_{f}{ }^{h}+\frac{1}{\Delta t} \mathcal{C} \Delta \tilde{p}_{f}^{h}+\mathcal{B} \tilde{p}_{f}^{h}=f_{2}
\end{aligned}
$$

where

$$
\begin{gathered}
\mathcal{M}=\frac{1}{Q} \int_{\Omega} \boldsymbol{N}^{p, T} \boldsymbol{N}^{p} d \Omega \\
\mathcal{H}=\frac{1}{g \rho_{w}} \int_{\Omega^{\oplus}} \boldsymbol{B}^{p, T} \cdot \boldsymbol{\kappa}^{\oplus} \boldsymbol{B}^{p} d \Omega+\frac{1}{g \rho_{w}} \int_{\Omega^{\ominus}} \boldsymbol{B}^{p, T} \cdot \boldsymbol{\kappa}^{\ominus} \boldsymbol{B}^{p} d \Omega \\
\mathcal{L}=\frac{1}{Q} \int_{\Omega^{\oplus}} \boldsymbol{N}^{p, T} M_{\mathrm{w}}^{p, \oplus} d \Omega+\frac{1}{Q} \int_{\Omega^{\ominus}} \boldsymbol{N}^{p, T} M_{\mathrm{w}}^{p, \ominus} d \Omega, \\
\mathcal{G}=\frac{1}{g \rho_{w}} \int_{\Omega^{\oplus}} \boldsymbol{B}^{p, T} \cdot \boldsymbol{\kappa}^{\oplus} \boldsymbol{G}_{\mathrm{w}}^{p, \oplus} d \Omega+\frac{1}{g \rho_{w}} \int_{\Omega^{\ominus}} \boldsymbol{B}^{p, T} \cdot \boldsymbol{\kappa}^{\ominus} \boldsymbol{G}_{\mathrm{w}}^{p, \ominus} d \Omega, \\
\boldsymbol{f}_{\mathbf{l e x t}_{\text {ext }}}=\int_{\Gamma_{q}} \boldsymbol{N}^{p, T} \bar{q}_{f} d \partial \Omega+\frac{1}{g} \int_{\Omega} \boldsymbol{B}^{p, T} \cdot \boldsymbol{\kappa} \boldsymbol{b} d \Omega, \\
\mathcal{C}=\frac{1}{Q} \int_{\Omega^{\oplus}} M_{\mathrm{w}}^{p, \oplus, T} M_{\mathrm{w}}^{p, \oplus} d \Omega+\frac{1}{Q} \int_{\Omega^{\ominus}} M_{\mathrm{w}}^{p, \ominus, T} M_{\mathrm{w}}^{p, \ominus} d \Omega,
\end{gathered}
$$




$$
\begin{gathered}
\mathcal{B}=\frac{1}{g \rho_{w}} \int_{\Omega^{\oplus}} \boldsymbol{G}_{\mathrm{w}}^{p, \oplus, T} \cdot \boldsymbol{\kappa}^{\oplus} \boldsymbol{G}_{\mathrm{w}}^{p, \oplus} d \Omega+\frac{1}{g \rho_{w}} \int_{\Omega^{\ominus}} \boldsymbol{G}_{\mathrm{w}}^{p, \ominus, T} \cdot \boldsymbol{\kappa}^{\ominus} \boldsymbol{G}_{\mathrm{w}}^{p, \ominus} d \Omega, \\
f_{2}=\int_{\Gamma_{q}} M_{\mathrm{w}}^{p, T} \bar{q}_{f} d \partial \Omega+\frac{1}{g} \int_{\Omega} \boldsymbol{G}_{\mathrm{w}}^{p, T} \cdot \boldsymbol{\kappa} \boldsymbol{b} d \Omega .
\end{gathered}
$$

Remark: As for the mechanical problem, note that the first equation of the problem (54) is global. It has to be solved for all the elements. On the contrary, the second one is local.

It has to be solved only for elements split into two parts. This remark is very important for the resolution process described latter.

\section{Hydro-mechanical coupled problem : Global-local solving process}

\subsection{System to be solved}

The hydro-mechanical coupled problem to be solved is obtained by combining (31), (38), (54) and (55). This leads to

$$
\begin{aligned}
& \boldsymbol{K}_{\boldsymbol{u u}} \overline{\boldsymbol{u}}^{h}+\boldsymbol{K}_{\boldsymbol{u w}}[|\varepsilon|]-\boldsymbol{K}_{\boldsymbol{u} \overline{\boldsymbol{p}}} \overline{\boldsymbol{p}}_{\boldsymbol{f}}^{h}-\boldsymbol{K}_{\boldsymbol{u} \tilde{p}} \tilde{p}_{f}^{h}=\boldsymbol{f}_{\boldsymbol{e x \boldsymbol { t }}} \\
& \boldsymbol{K}_{\boldsymbol{u} \mathrm{w}}^{T} \overline{\boldsymbol{u}}^{h}+\boldsymbol{K}_{\mathrm{ww}}[|\varepsilon|]-\boldsymbol{K}_{\mathrm{w} \overline{\boldsymbol{p}}} \overline{\boldsymbol{p}}_{\boldsymbol{f}}^{h}-\boldsymbol{K}_{\mathrm{w} \tilde{p}} \tilde{p}_{f}^{h}=\mathbf{0}, \\
& \frac{1}{\Delta t} \boldsymbol{K}_{\boldsymbol{u} \overline{\boldsymbol{p}}}^{T} \Delta \overline{\boldsymbol{u}}^{h}+\frac{1}{\Delta t} \boldsymbol{K}_{\mathrm{w} \overline{\boldsymbol{p}}}^{T} \Delta[|\boldsymbol{\varepsilon}|]+\frac{1}{\Delta t} \mathcal{M} \Delta \overline{\boldsymbol{p}}_{\boldsymbol{f}}{ }^{h}+\mathcal{H} \overline{\boldsymbol{p}}_{\boldsymbol{f}}{ }^{h}+\frac{1}{\Delta t} \mathcal{L} \Delta \tilde{p}_{f}^{h}+\mathcal{G} \tilde{p}_{f}^{h}=\boldsymbol{f}_{\mathbf{1}_{\boldsymbol{e x t}}}, \\
& \frac{1}{\Delta t} \boldsymbol{K}_{\boldsymbol{u} \tilde{p}}^{T} \Delta \overline{\boldsymbol{u}}^{h}+\frac{1}{\Delta t} \boldsymbol{K}_{\mathrm{w} \tilde{p}}^{T} \Delta[|\varepsilon|]+\frac{1}{\Delta t} \mathcal{L}^{T} \Delta \overline{\boldsymbol{p}}_{\boldsymbol{f}}{ }^{h}+\mathcal{G}^{T} \overline{\boldsymbol{p}}_{\boldsymbol{f}}{ }^{h}+\frac{1}{\Delta t} \mathcal{C} \Delta \tilde{p}_{f}^{h}+\mathcal{B} \tilde{p}_{f}^{h}=f_{2},
\end{aligned}
$$

where the unknowns are $\overline{\boldsymbol{u}}^{h}, \overline{\boldsymbol{p}}_{\boldsymbol{f}}{ }^{h},[|\varepsilon|]$ and $\tilde{p}_{f}^{h}$.

The solving process is based upon the key idea of [28]. Since the residual equations (64b) and (64d) hold only for each element $e=1,2, \ldots, n_{\text {elm }}^{\oplus, \ominus}$ split into two parts, the most effective solution is to condense out the internal element modes $[|\varepsilon|]$ and $\tilde{p}_{f}^{h}$ to arrive at an equivalent displacement model involving only the nodal displacement vector $\overline{\boldsymbol{u}}^{h}$ at the global level, where the nodal pressure vector $\overline{\boldsymbol{p}}_{\boldsymbol{f}}{ }^{h}$ is then post- calculated.

The problem is therefore split into two levels of equations : (1) the global one corresponding to (64a) and (64c), (2) the local one corresponding to (64b) and (64d $)$. The term 
global means that the equations are applied to all the elements of the problem. The term local means that the equations have to be solved only for the elements $e=1,2, \ldots, n_{\text {elm }}^{\oplus, \ominus}$ split into two parts. Due to the fact that the E-FEM method consists in adding new unknowns $\left([|\varepsilon|]\right.$ and $\tilde{p}_{f}^{h}$ in this case) at the element level, the value of these unknowns is discontinuous passing through the boundaries of the elements and so this local property allows a static condensation ([34]) once these new unknowns are solved.

In the rest of this paper and technically speaking, these two levels of equations global and local - correspond to indices called respectively $k$ and $l$. For a given iterate $\left.\Delta \overline{\boldsymbol{u}}^{h}\right|_{n+1} ^{(k)},\left.\Delta \overline{\boldsymbol{p}}_{\boldsymbol{f}}\right|_{n+1} ^{(k)}$, we perform a local sub-iteration on $l$ to solve exactly the non-linear equations (64b) and (64d).

In order to solve the system, one has first to linearize it. This is explained is the next four subsections.

\subsection{Linearization of $64 a$}

Let's call the left hand side of (64a), $\boldsymbol{f}_{\text {int }}$ thus we have at the $k$ th iteration at the time step $n+1$

$$
\left[\left.\boldsymbol{f}_{\boldsymbol{i n t}}\left(\overline{\boldsymbol{u}}^{h},[|\varepsilon|], \overline{\boldsymbol{p}}_{\boldsymbol{f}}^{h}, \tilde{p}_{f}^{h}\right)\right|_{n+1} ^{(k)}-\left.\boldsymbol{f}_{\text {ext }}\right|_{n+1}\right]=\mathbf{0} .
$$

The linearization of (65) at the global level corresponding to $k$ yields

$$
\begin{aligned}
& {\left[\left.\boldsymbol{f}_{\boldsymbol{i n t}}\left(\overline{\boldsymbol{u}}^{h},[|\boldsymbol{\varepsilon}|], \overline{\boldsymbol{p}}_{\boldsymbol{f}}^{h}, \tilde{p}_{f}^{h}\right)\right|_{n+1} ^{(k+1)}-\left.\boldsymbol{f}_{\text {ext }}\right|_{n+1}\right]=} \\
& {\left[\left.\boldsymbol{f}_{\boldsymbol{i n \boldsymbol { t }}}\left(\overline{\boldsymbol{u}}^{h},[|\boldsymbol{\varepsilon}|], \overline{\boldsymbol{p}}_{\boldsymbol{f}}^{h}, \tilde{p}_{f}^{h}\right)\right|_{n+1} ^{(k)}-\left.\boldsymbol{f}_{\text {ext }}\right|_{n+1}\right]+\left.\left.\frac{\partial \boldsymbol{f}_{\boldsymbol{i n t}}(X)}{\partial X}\right|_{n+1} ^{(k)} \Delta X\right|_{n+1} ^{(k+1)} \simeq \mathbf{0},}
\end{aligned}
$$

where

$$
\begin{aligned}
\left.\left.\frac{\partial \boldsymbol{f}_{\boldsymbol{i n t}}(X)}{\partial X}\right|_{n+1} ^{(k)} \Delta X\right|_{n+1} ^{(k+1)} & =\left.\left.\boldsymbol{K}_{\boldsymbol{u u}}\right|_{n+1} ^{(k)} \Delta \overline{\boldsymbol{u}}^{h}\right|_{n+1} ^{(k+1)}+\left.\left.\boldsymbol{K}_{\boldsymbol{u w}}\right|_{n+1} ^{(k)} \Delta[|\varepsilon|]\right|_{n+1} ^{(k+1)} \\
& -\left.\left.\boldsymbol{K}_{\boldsymbol{u} \overline{\boldsymbol{p}}}\right|_{n+1} ^{(k)} \Delta \overline{\boldsymbol{p}}_{\boldsymbol{f}}^{h}\right|_{n+1} ^{(k+1)}-\left.\left.\boldsymbol{K}_{\boldsymbol{u} \tilde{p}}\right|_{n+1} ^{(k)} \Delta \tilde{p}_{f}^{h}\right|_{n+1} ^{(k+1)}
\end{aligned}
$$


Finally one gets

$$
\begin{aligned}
& \left.\left.\boldsymbol{K}_{\boldsymbol{u u}}\right|_{n+1} ^{(k)} \Delta \overline{\boldsymbol{u}}^{h}\right|_{n+1} ^{(k+1)}+\left.\left.\boldsymbol{K}_{\boldsymbol{u w}}\right|_{n+1} ^{(k)} \Delta[|\boldsymbol{\varepsilon}|]\right|_{n+1} ^{(k+1)}-\left.\left.\boldsymbol{K}_{\boldsymbol{u} \overline{\boldsymbol{p}}}\right|_{n+1} ^{(k)} \Delta \overline{\boldsymbol{p}}_{\boldsymbol{f}}^{h}\right|_{n+1} ^{(k+1)}-\left.\left.\boldsymbol{K}_{\boldsymbol{u} \tilde{p}}\right|_{n+1} ^{(k)} \Delta \tilde{p}_{f}^{h}\right|_{n+1} ^{(k+1)} \\
& =-\left[\left.\boldsymbol{f}_{\boldsymbol{i n t}}\left(\overline{\boldsymbol{u}}^{h},[|\boldsymbol{\varepsilon}|], \overline{\boldsymbol{p}}_{\boldsymbol{f}}^{h}, \tilde{p}_{f}^{h}\right)\right|_{n+1} ^{(k)}-\left.\boldsymbol{f}_{\text {ext }}\right|_{n+1}\right] .
\end{aligned}
$$

\subsection{Linearization of 64 c}

Let's call the left hand side of ([64c), $\boldsymbol{f}_{\mathbf{1}_{\text {int }}}$ thus we have at the $k$ th iteration at the time step $n+1$

$$
\left[\left.\boldsymbol{f}_{\mathbf{1 i n t}}\left(\Delta \overline{\boldsymbol{u}}^{h}, \Delta[|\varepsilon|], \overline{\boldsymbol{p}}_{\boldsymbol{f}}^{h}, \Delta \overline{\boldsymbol{p}}_{\boldsymbol{f}}{ }^{h}, \tilde{p}_{f}^{h}, \Delta \tilde{p}_{f}^{h}\right)\right|_{n+1} ^{(k)}-\left.\boldsymbol{f}_{\mathbf{1} \boldsymbol{e x t}}\right|_{n+1}\right]=\mathbf{0} .
$$

The linearization of (69) at the global level corresponding to $k$ yields

$$
\begin{aligned}
& {\left[\left.\boldsymbol{f}_{\boldsymbol{1}_{\text {int }}}\left(\Delta \overline{\boldsymbol{u}}^{h}, \Delta[|\boldsymbol{\varepsilon}|], \overline{\boldsymbol{p}}_{\boldsymbol{f}}{ }^{h}, \Delta \overline{\boldsymbol{p}}_{\boldsymbol{f}}{ }^{h}, \tilde{p}_{f}^{h}, \Delta \tilde{p}_{f}^{h}\right)\right|_{n+1} ^{(k+1)}-\boldsymbol{f}_{\left.\mathbf{1}_{\boldsymbol{e x t}}\right|_{n+1}}\right]=} \\
& {\left[\left.\boldsymbol{f}_{\mathbf{1}_{\boldsymbol{i n t}}}\left(\Delta \overline{\boldsymbol{u}}^{h}, \Delta[|\boldsymbol{\varepsilon}|], \overline{\boldsymbol{p}}_{\boldsymbol{f}}^{h}, \Delta \overline{\boldsymbol{p}}_{\boldsymbol{f}}{ }^{h}, \tilde{p}_{f}^{h}, \Delta \tilde{p}_{f}^{h}\right)\right|_{n+1} ^{(k)}-\left.\boldsymbol{f}_{\mathbf{1}_{\boldsymbol{e x t}}}\right|_{n+1}\right]+\left.\left.\frac{\partial \boldsymbol{f}_{\mathbf{1}_{\text {int }}}(X)}{\partial X}\right|_{n+1} ^{(k)} \Delta X\right|_{n+1} ^{(k+1)} \simeq \mathbf{0},}
\end{aligned}
$$

where

$$
\begin{aligned}
\left.\left.\frac{\partial \boldsymbol{f}_{\mathbf{1}_{\boldsymbol{i n} \boldsymbol{t}}}(X)}{\partial X}\right|_{n+1} ^{(k)} \Delta X\right|_{n+1} ^{(k+1)} & =\left.\left.\frac{1}{\Delta t} \boldsymbol{K}_{\boldsymbol{u} \overline{\boldsymbol{p}}}^{T}\right|_{n+1} ^{(k)} \Delta \overline{\boldsymbol{u}}^{h}\right|_{n+1} ^{(k+1)}+\left.\frac{1}{\Delta t} \boldsymbol{K}_{\mathrm{w} \overline{\boldsymbol{p}}}^{T} \Delta[|\boldsymbol{\varepsilon}|]\right|_{n+1} ^{(k+1)} \\
& +\left.\left.\left[\frac{1}{\Delta t} \boldsymbol{\mathcal { M }}+\mathcal{H}\right]\right|_{n+1} ^{(k)} \Delta \overline{\boldsymbol{p}}_{\boldsymbol{f}}^{h}\right|_{n+1} ^{(k+1)}+\left.\left.\left[\frac{1}{\Delta t} \mathcal{L}+\mathcal{G}\right]\right|_{n+1} ^{(k)} \Delta \tilde{p}_{f}^{h}\right|_{n+1} ^{(k+1)}
\end{aligned}
$$

Finally one obtains

$$
\begin{aligned}
& \left.\left.\frac{1}{\Delta t} \boldsymbol{K}_{\boldsymbol{u} \overline{\boldsymbol{p}}}^{T}\right|_{n+1} ^{(k)} \Delta \overline{\boldsymbol{u}}^{h}\right|_{n+1} ^{(k+1)}+\left.\frac{1}{\Delta t} \boldsymbol{K}_{\mathrm{w} \overline{\boldsymbol{p}}}^{T} \Delta[|\boldsymbol{\varepsilon}|]\right|_{n+1} ^{(k+1)}+\left.\left.\left[\frac{1}{\Delta t} \boldsymbol{\mathcal { M }}+\mathcal{H}\right]\right|_{n+1} ^{(k)} \Delta \overline{\boldsymbol{p}}_{\boldsymbol{f}}^{h}\right|_{n+1} ^{(k+1)} \\
& +\left.\left.\left[\frac{1}{\Delta t} \mathcal{L}+\mathcal{G}\right]\right|_{n+1} ^{(k)} \Delta \tilde{p}_{f}^{h}\right|_{n+1} ^{(k+1)}=-\left[\left.\boldsymbol{f}_{\mathbf{1}_{\boldsymbol{i n t}}}\left(\Delta \overline{\boldsymbol{u}}^{h}, \Delta[|\boldsymbol{\varepsilon}|], \overline{\boldsymbol{p}}_{\boldsymbol{f}}^{h}, \Delta \overline{\boldsymbol{p}}_{\boldsymbol{f}}^{h}, \tilde{p}_{f}^{h}, \Delta \tilde{p}_{f}^{h}\right)\right|_{n+1} ^{(k)}-\boldsymbol{f}_{\left.\mathbf{1}_{\text {ext }}\right|_{n+1}}\right] .
\end{aligned}
$$

\subsection{Linearization of 64b}

Let's call ([64b), $\boldsymbol{h}_{e}\left(\overline{\boldsymbol{u}}^{h},[|\varepsilon|], \overline{\boldsymbol{p}}_{f}{ }^{h}, \tilde{p}_{f}^{h}\right)$. The linearization of ([64b) is performed localy for a given global iterate $k$. In other word, for a given $k$, a local sub-iteration on $l$ is done in order to solve exactly (64b). 
For a given $k$ and an iterate $l$, we have

$\left.\boldsymbol{h}_{e}\left(\overline{\boldsymbol{u}}^{h},[|\boldsymbol{\varepsilon}|], \overline{\boldsymbol{p}}_{\boldsymbol{f}}^{h}, \tilde{p}_{f}^{h}\right)\right|_{n+1} ^{(k, l)}=\left.\boldsymbol{K}_{\boldsymbol{u} \mathrm{w}}^{T} \overline{\boldsymbol{u}}^{h}\right|_{n+1} ^{(k)}+\left.\boldsymbol{K}_{\mathrm{ww}}[|\varepsilon|]\right|_{n+1} ^{(k, l)}-\left.\boldsymbol{K}_{\mathrm{w} \overline{\boldsymbol{p}}} \overline{\boldsymbol{p}}_{\boldsymbol{f}}^{h}\right|_{n+1} ^{(k)}-\left.\boldsymbol{K}_{\mathrm{w} \tilde{p}} \tilde{p}_{f}^{h}\right|_{n+1} ^{(k, l)}=\mathbf{0}$.

This can be rewritten with the increment of the unknowns under the form

$$
\begin{aligned}
& \boldsymbol{K}_{\boldsymbol{u w}}{ }^{T}\left[\left.\overline{\boldsymbol{u}}^{h}\right|_{n}+\left.\Delta \overline{\boldsymbol{u}}^{h}\right|_{n+1} ^{(k)}\right]+\boldsymbol{K}_{\mathrm{ww}}\left[\left.[|\boldsymbol{\varepsilon}|]\right|_{n}+\left.\Delta[|\varepsilon|]\right|_{n+1} ^{(k, l)}\right]-\boldsymbol{K}_{\mathrm{w} \overline{\boldsymbol{p}}}\left[\left.\overline{\boldsymbol{p}}_{\boldsymbol{f}}{ }^{h}\right|_{n}+\left.\left.\Delta \overline{\boldsymbol{p}}_{\boldsymbol{f}}\right|^{(k)}\right|_{n+1} ^{(k)}\right] \\
& -\boldsymbol{K}_{\mathrm{w} \tilde{p}}\left[\left.\tilde{p}_{f}^{h}\right|_{n}+\left.\Delta \tilde{p}_{f}^{h}\right|_{n+1} ^{(k, l)}\right]=\mathbf{0} .
\end{aligned}
$$

After collecting terms together, one gets

$$
\begin{aligned}
& \left.\boldsymbol{K}_{\boldsymbol{u} \mathrm{w}}{ }^{T} \overline{\boldsymbol{u}}^{h}\right|_{n}+\left.\boldsymbol{K}_{\mathrm{ww}}[|\varepsilon|]\right|_{n}-\left.\boldsymbol{K}_{\mathrm{w} \overline{\boldsymbol{p}}} \overline{\boldsymbol{p}}_{\boldsymbol{f}}^{h}\right|_{n}-\left.\boldsymbol{K}_{\mathrm{w} \tilde{p}} \tilde{p}_{f}^{h}\right|_{n}+\left.\boldsymbol{K}_{\boldsymbol{u} \mathrm{w}}{ }^{T} \Delta \overline{\boldsymbol{u}}^{h}\right|_{n+1} ^{(k)} \\
& +\left.\boldsymbol{K}_{\mathrm{ww}} \Delta[|\varepsilon|]\right|_{n+1} ^{(k, l)}-\left.\boldsymbol{K}_{\mathrm{w} \overline{\boldsymbol{p}}} \Delta \overline{\boldsymbol{p}}_{\boldsymbol{f}}{ }^{h}\right|_{n+1} ^{(k)}-\left.\boldsymbol{K}_{\mathrm{w} \tilde{p}} \Delta \tilde{p}_{f}^{h}\right|_{n+1} ^{(k, l)}=\mathbf{0}
\end{aligned}
$$

or under a pithy form

$$
\begin{aligned}
& \left.\boldsymbol{h}_{e}\left(\overline{\boldsymbol{u}}^{h},[|\varepsilon|], \overline{\boldsymbol{p}}_{\boldsymbol{f}}^{h}, \tilde{p}_{f}^{h}\right)\right|_{n}+\left.\boldsymbol{K}_{\boldsymbol{u \mathrm { w }}}^{T} \Delta \overline{\boldsymbol{u}}^{h}\right|_{n+1} ^{(k)}+\left.\boldsymbol{K}_{\mathrm{ww}} \Delta[|\boldsymbol{\varepsilon}|]\right|_{n+1} ^{(k, l)}-\left.\boldsymbol{K}_{\mathrm{w} \overline{\boldsymbol{p}}} \Delta \overline{\boldsymbol{p}}_{\boldsymbol{f}}{ }^{h}\right|_{n+1} ^{(k)} \\
& -\left.\boldsymbol{K}_{\mathrm{w} \tilde{p}} \Delta \tilde{p}_{f}^{h}\right|_{n+1} ^{(k, l)}=\mathbf{0} .
\end{aligned}
$$

Introducing a trial function $\left.\boldsymbol{h}_{e}\left(\overline{\boldsymbol{u}}^{h},[|\varepsilon|], \overline{\boldsymbol{p}}_{\boldsymbol{f}}^{h}, \tilde{p}_{f}^{h}\right)\right|_{n+1} ^{\text {trial }}$ in the spirit of a return mapping algorithm $([35])$ such as

$$
\left.\boldsymbol{h}_{e}\left(\overline{\boldsymbol{u}}^{h},[|\boldsymbol{\varepsilon}|], \overline{\boldsymbol{p}}_{\boldsymbol{f}}^{h}, \tilde{p}_{f}^{h}\right)\right|_{n+1} ^{\text {trial }}=\left.\boldsymbol{h}_{e}\left(\overline{\boldsymbol{u}}^{h},[|\varepsilon|], \overline{\boldsymbol{p}}_{\boldsymbol{f}}^{h}, \tilde{p}_{f}^{h}\right)\right|_{n}+\left.\boldsymbol{K}_{\boldsymbol{u w}}^{T} \Delta \overline{\boldsymbol{u}}^{h}\right|_{n+1} ^{(k)}-\left.\boldsymbol{K}_{\mathrm{w} \overline{\boldsymbol{p}}} \Delta \overline{\boldsymbol{p}}_{\boldsymbol{f}}{ }^{h}\right|_{n+1} ^{(k)}
$$

one gets

$$
\left.\boldsymbol{h}_{e}\left(\overline{\boldsymbol{u}}^{h},[|\varepsilon|], \overline{\boldsymbol{p}}_{\boldsymbol{f}}^{h}, \tilde{p}_{f}^{h}\right)\right|_{n+1} ^{\text {trial }}+\left.\boldsymbol{K}_{\mathrm{ww}} \Delta[|\varepsilon|]\right|_{n+1} ^{(k, l)}-\left.\boldsymbol{K}_{\mathrm{w} \tilde{p}} \Delta \tilde{p}_{f}^{h}\right|_{n+1} ^{(k, l)}=\mathbf{0} .
$$

(78) has to be linearized in relation with $l$, this yields

$$
\left.\boldsymbol{h}_{e}\left(\overline{\boldsymbol{u}}^{h},[|\boldsymbol{\varepsilon}|], \overline{\boldsymbol{p}}_{\boldsymbol{f}}^{h}, \tilde{p}_{f}^{h}\right)\right|_{n+1} ^{(k, l+1)}=\left.\boldsymbol{h}_{e}\left(\overline{\boldsymbol{u}}^{h},[|\boldsymbol{\varepsilon}|], \overline{\boldsymbol{p}}_{\boldsymbol{f}}^{h}, \tilde{p}_{f}^{h}\right)\right|_{n+1} ^{(k, l)}+\left.\left.\frac{\partial \boldsymbol{h}_{e}(\Delta X)}{\partial \Delta X}\right|_{n+1} ^{(k, l)} \delta \Delta X\right|_{n+1} ^{(k, l+1)} \simeq \mathbf{0}
$$

where

$$
\left.\left.\frac{\partial \boldsymbol{h}_{e}(\Delta X)}{\partial \Delta X}\right|_{n+1} ^{(k, l)} \delta \Delta X\right|_{n+1} ^{(k, l+1)}=\left.\left.\boldsymbol{K}_{\mathrm{ww}}\right|_{n+1} ^{(k, l)} \delta \Delta[|\varepsilon|]\right|_{n+1} ^{(k, l+1)}-\left.\left.\boldsymbol{K}_{\mathrm{w} \tilde{p}}\right|_{n+1} ^{(k, l)} \delta \Delta \tilde{p}_{f}^{h}\right|_{n+1} ^{(k, l+1)} .
$$


Finally one gets

$$
\left.\left.\boldsymbol{K}_{\mathrm{ww}}\right|_{n+1} ^{(k, l)} \delta \Delta[|\varepsilon|]\right|_{n+1} ^{(k, l+1)}-\left.\left.\boldsymbol{K}_{\mathrm{w} \tilde{p}}\right|_{n+1} ^{(k, l)} \delta \Delta \tilde{p}_{f}^{h}\right|_{n+1} ^{(k, l+1)}=-\left.\boldsymbol{h}_{e}\left(\overline{\boldsymbol{u}}^{h},[|\varepsilon|], \overline{\boldsymbol{p}}_{\boldsymbol{f}}^{h}, \tilde{p}_{f}^{h}\right)\right|_{n+1} ^{(k, l)}
$$

\subsection{Linearization of $64 \mathrm{~d}$}

Let's call ([64d), $s_{e}\left(\Delta \overline{\boldsymbol{u}}^{h}, \Delta[|\boldsymbol{\varepsilon}|], \overline{\boldsymbol{p}}_{\boldsymbol{f}}^{h}, \Delta \overline{\boldsymbol{p}}_{\boldsymbol{f}}{ }^{h}, \tilde{p}_{f}^{h}, \Delta \tilde{p}_{f}^{h}\right)$. The linearization of (64d) is performed localy for a given global iterate $k$. In other word, for a given $k$, a local sub-iteration on $l$ is done in order to solve exactly (64d).

For a given $k$ and an iterate $l$, we have (neglecting $f_{2}$ )

$$
\begin{aligned}
& \left.s_{e}\left(\Delta \overline{\boldsymbol{u}}^{h}, \Delta[|\boldsymbol{\varepsilon}|], \overline{\boldsymbol{p}}_{\boldsymbol{f}}^{h}, \Delta \overline{\boldsymbol{p}}_{\boldsymbol{f}}^{h}, \tilde{p}_{f}^{h}, \Delta \tilde{p}_{f}^{h}\right)\right|_{n+1} ^{(k, l)}=\left.\frac{1}{\Delta t} \boldsymbol{K}_{\boldsymbol{u} \tilde{p}}^{T} \Delta \overline{\boldsymbol{u}}^{h}\right|_{n+1} ^{(k)}+\left.\frac{1}{\Delta t} \boldsymbol{K}_{\mathrm{w} \tilde{p}}^{T} \Delta[|\varepsilon|]\right|_{n+1} ^{(k, l)} \\
& +\left.\frac{1}{\Delta t} \mathcal{L}^{T} \Delta \overline{\boldsymbol{p}}_{\boldsymbol{f}}\right|_{n+1} ^{(k)}+\left.\boldsymbol{\mathcal { G }}^{T} \overline{\boldsymbol{p}}_{\boldsymbol{f}}\right|_{n+1} ^{(k)}+\left.\frac{1}{\Delta t} \mathcal{C} \Delta \tilde{p}_{f}^{h}\right|_{n+1} ^{(k, l)}+\left.\mathcal{B} \tilde{p}_{f}^{h}\right|_{n+1} ^{(k, l)}=0 .
\end{aligned}
$$

Following the same reasoning as for (64b) , one gets

$$
\left.s_{e}\left(\Delta \overline{\boldsymbol{u}}^{h}, \Delta[|\varepsilon|], \overline{\boldsymbol{p}}_{\boldsymbol{f}}^{h}, \Delta \overline{\boldsymbol{p}}_{\boldsymbol{f}}{ }^{h}, \tilde{p}_{f}^{h}, \Delta \tilde{p}_{f}^{h}\right)\right|_{n+1} ^{\text {trial }}+\left.\frac{1}{\Delta t} \boldsymbol{K}_{\mathrm{w} \tilde{p}}^{T} \Delta[|\varepsilon|]\right|_{n+1} ^{(k, l)}+\left.\left[\frac{1}{\Delta t} \mathcal{C}+\mathcal{B}\right] \Delta \tilde{p}_{f}^{h}\right|_{n+1} ^{(k, l)}=\mathbf{0},
$$

where

$$
\begin{aligned}
& \left.s_{e}\left(\Delta \overline{\boldsymbol{u}}^{h}, \Delta[|\boldsymbol{\varepsilon}|], \overline{\boldsymbol{p}}_{\boldsymbol{f}}^{h}, \Delta \overline{\boldsymbol{p}}_{\boldsymbol{f}}{ }^{h}, \tilde{p}_{f}^{h}, \Delta \tilde{p}_{f}^{h}\right)\right|_{n+1} ^{\text {trial }}=\left.s_{e}\left(\Delta \overline{\boldsymbol{u}}^{h}, \Delta[|\boldsymbol{\varepsilon}|], \overline{\boldsymbol{p}}_{\boldsymbol{f}}^{h}, \Delta \overline{\boldsymbol{p}}_{\boldsymbol{f}}{ }^{h}, \tilde{p}_{f}^{h}, \Delta \tilde{p}_{f}^{h}\right)\right|_{n} \\
& +\left.\frac{1}{\Delta t} \boldsymbol{K}_{\boldsymbol{u} \tilde{p}}^{T} \Delta \overline{\boldsymbol{u}}^{h}\right|_{n+1} ^{(k)}+\left.\left[\frac{1}{\Delta t} \mathcal{L}^{T}+\mathcal{G}^{T}\right] \Delta \overline{\boldsymbol{p}}_{\boldsymbol{f}}\right|_{n+1} ^{(k)} .
\end{aligned}
$$

After linearizing (83), one obtains

$$
\begin{aligned}
& \left.\left.\frac{1}{\Delta t} \boldsymbol{K}_{\mathrm{w} \tilde{p}}^{T}\right|_{n+1} ^{(k, l)} \delta \Delta[|\varepsilon|]\right|_{n+1} ^{(k, l+1)}+\left.\left.\left[\frac{1}{\Delta t} \mathcal{C}+\mathcal{B}\right]\right|_{n+1} ^{(k, l)} \delta \Delta \tilde{p}_{f}^{h}\right|_{n+1} ^{(k, l+1)} \\
& =-\left.s_{e}\left(\Delta \overline{\boldsymbol{u}}^{h}, \Delta[|\boldsymbol{\varepsilon}|], \overline{\boldsymbol{p}}_{f}^{h}, \Delta \overline{\boldsymbol{p}}_{\boldsymbol{f}}{ }^{h}, \tilde{p}_{f}^{h}, \Delta \tilde{p}_{f}^{h}\right)\right|_{n+1} ^{(k, l)}
\end{aligned}
$$

Having the linearization of the coupled system in hands, we now turn to its resolution. As mentioned above, this is achieved at two levels : a local one and a global one. 


\subsection{Algorithm for the local system resolution}

The local system to be solved is

$$
\begin{aligned}
& \left.\left.\boldsymbol{K}_{\mathrm{ww}}\right|_{n+1} ^{(k, l)} \delta \Delta[|\varepsilon|]\right|_{n+1} ^{(k, l+1)}-\left.\left.\boldsymbol{K}_{\mathrm{w} \tilde{p}}\right|_{n+1} ^{(k, l)} \delta \Delta \tilde{p}_{f}^{h}\right|_{n+1} ^{(k, l+1)}=-\left.\boldsymbol{h}_{e}\left(\overline{\boldsymbol{u}}^{h},[|\boldsymbol{\varepsilon}|], \overline{\boldsymbol{p}}_{\boldsymbol{f}}^{h}, \tilde{p}_{f}^{h}\right)\right|_{n+1} ^{(k, l)}, \\
& \left.\left.\frac{1}{\Delta t} \boldsymbol{K}_{\mathrm{w} \tilde{p}}^{T}\right|_{n+1} ^{(k, l)} \delta \Delta[|\varepsilon|]\right|_{n+1} ^{(k, l+1)}+\left.\left.\left[\frac{1}{\Delta t} \mathcal{C}+\mathcal{B}\right]\right|_{n+1} ^{(k, l)} \delta \Delta \tilde{p}_{f}^{h}\right|_{n+1} ^{(k, l+1)} \\
& =-\left.s_{e}\left(\Delta \overline{\boldsymbol{u}}^{h}, \Delta[|\varepsilon|], \overline{\boldsymbol{p}}_{\boldsymbol{f}}{ }^{h}, \Delta \overline{\boldsymbol{p}}_{\boldsymbol{f}}{ }^{h}, \tilde{p}_{f}^{h}, \Delta \tilde{p}_{f}^{h}\right)\right|_{n+1} ^{(k, l)},
\end{aligned}
$$

in terms of $\delta \Delta \tilde{p}_{f}^{h}$ and $\delta \Delta[|\varepsilon|]$. We remind that this system has to be solved only for the elements split into two parts.

After a little work, the solution of this system is

$$
\begin{aligned}
&\left.\delta \Delta[|\varepsilon|]\right|_{n+1} ^{(k, l+1)}=-\left.\left[\boldsymbol{K}_{\mathrm{ww}}+\frac{1}{\Delta t\left[\frac{1}{\Delta t} \mathcal{C}+\mathcal{B}\right]} \boldsymbol{K}_{\mathrm{w} \tilde{p}} \boldsymbol{K}_{\mathrm{w} \tilde{p}}^{T}\right]^{-1}\left[\boldsymbol{h}_{e}+\frac{s_{e}}{\left[\frac{1}{\Delta t} \mathcal{C}+\mathcal{B}\right]} \boldsymbol{K}_{\mathrm{w} \tilde{p}}\right]\right|_{n+1} ^{(k, l)} \\
&\left.\delta \Delta \tilde{p}_{f}^{h}\right|_{n+1} ^{(k, l+1)}= {\left[-\frac{s_{e}}{\left[\frac{1}{\Delta t} \mathcal{C}+\mathcal{B}\right]}+\frac{1}{\Delta t\left[\frac{1}{\Delta t} \mathcal{C}+\mathcal{B}\right]} \boldsymbol{K}_{\mathrm{w} \tilde{p}}^{T}\left[\boldsymbol{K}_{\mathrm{ww}}+\frac{1}{\Delta t\left[\frac{1}{\Delta t} \mathcal{C}+\mathcal{B}\right]} \boldsymbol{K}_{\mathrm{w} \tilde{p}} \boldsymbol{K}_{\mathrm{w} \tilde{p}}^{T}\right]^{-1}\right.} \\
& {\left.\left[\boldsymbol{h}_{e}+\frac{s_{e}}{\left[\frac{1}{\Delta t} \mathcal{C}+\mathcal{B}\right]} \boldsymbol{K}_{\mathrm{w} \tilde{p}]}\right]\right|_{n+1} ^{(k, l)} }
\end{aligned}
$$

This local system resolution is performed in the more general case when $\left.\boldsymbol{h}_{e}\right|_{n+1} ^{\text {trial }} \neq \mathbf{0}$ and $\left.s_{e}\right|_{n+1} ^{\text {trial }} \neq 0$; in this case algorithm (11) is performed. Intermediate cases exist but they are not detailed at this stage.

Once convergence is achieved, $\Delta[|\varepsilon|]$ and $\Delta \tilde{p}_{f}^{h}$ are known.

The next subsection presents the global system resolution strategy in the spirit of [35].

\subsection{Global system resolution strategy}

We let $\left.(\bullet)\right|_{n+1} ^{(k)}$ be the value of a variable $(\bullet)$ at the $k$ th iteration during the load step in $\left[t_{n}, t_{n+1}\right]$. Accordingly,

i) Let $\left.\Delta \overline{\boldsymbol{u}}^{h}\right|_{n+1} ^{(k)}$ and $\left.\Delta \overline{\boldsymbol{p}}_{\boldsymbol{f}}\right|_{n+1} ^{(k)}$ be the incremental nodal displacement and pressure at the $k$ th iteration and let

$$
\begin{gathered}
\left.\overline{\boldsymbol{u}}^{h}\right|_{n+1} ^{(k)}=\left.\overline{\boldsymbol{u}}^{h}\right|_{n}+\left.\Delta \overline{\boldsymbol{u}}^{h}\right|_{n+1} ^{(k)}, \\
\left.\overline{\boldsymbol{p}}_{\boldsymbol{f}}\right|^{(k)}=\left.\overline{\boldsymbol{p}}_{\boldsymbol{f}}^{h}\right|_{n}+\left.\left.\Delta \overline{\boldsymbol{p}}_{\boldsymbol{f}}\right|^{(k)}\right|_{n+1} ^{(k)},
\end{gathered}
$$




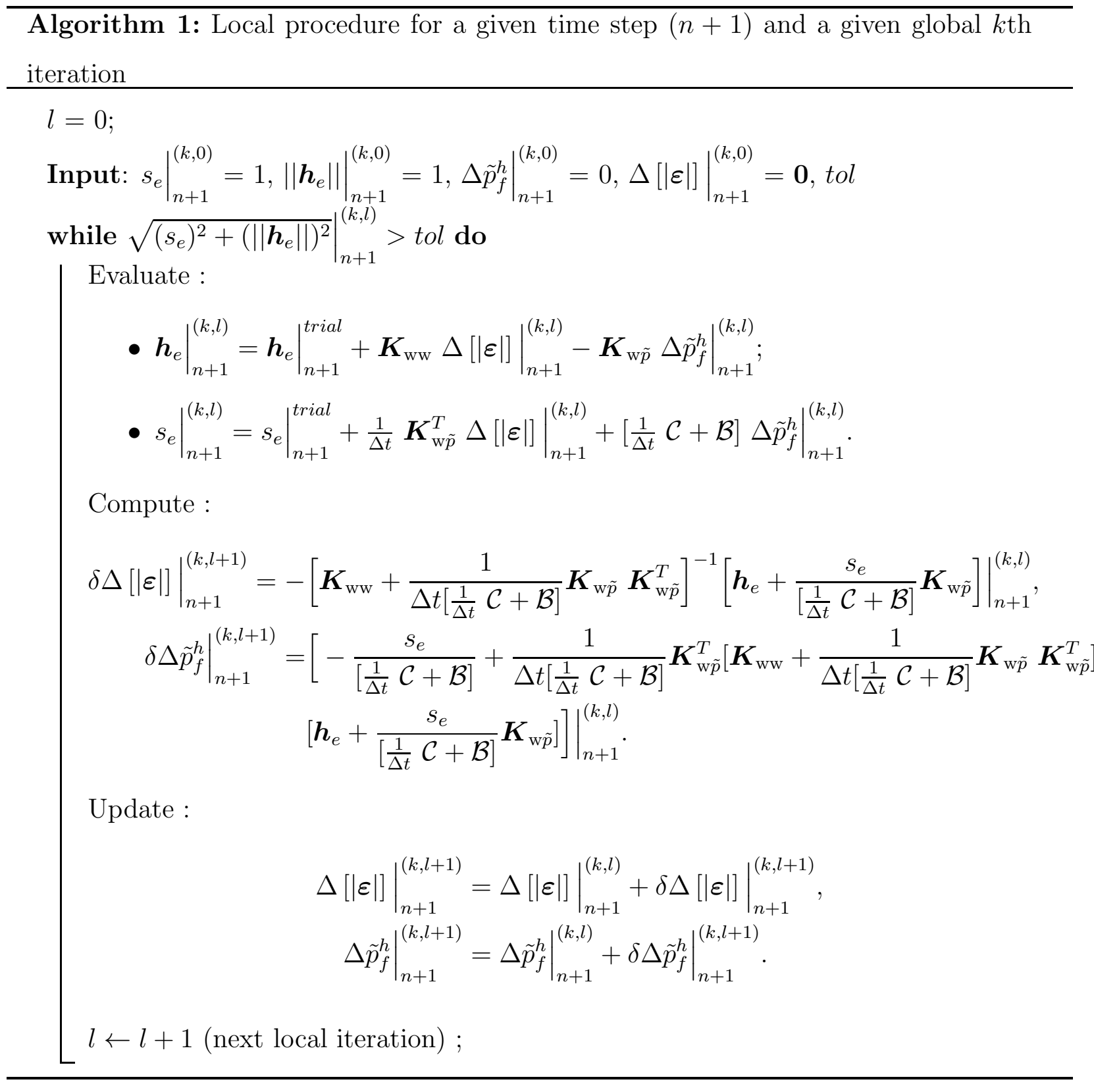


be the total nodal displacement and pressure;

ii) Freeze $\left.\Delta \overline{\boldsymbol{u}}^{h}\right|_{n+1} ^{(k)}$ and $\left.\Delta \overline{\boldsymbol{p}}_{\boldsymbol{f}}\right|_{n+1} ^{(k)}$ and compute $\left.\Delta[|\boldsymbol{\varepsilon}|]\right|_{n+1} ^{(k)}$ and $\left.\Delta \tilde{p}_{f}^{h}\right|_{n+1} ^{(k)}$ thanks to algorithm (1) and let

$$
\begin{gathered}
{\left.[|\varepsilon|]\right|_{n+1} ^{(k)}=\left.[|\varepsilon|]\right|_{n}+\left.\Delta[|\varepsilon|]\right|_{n+1} ^{(k)},} \\
\left.\tilde{p}_{f}^{h}\right|_{n+1} ^{(k)}=\left.\tilde{p}_{f}^{h}\right|_{n}+\left.\Delta \tilde{p}_{f}^{h}\right|_{n+1} ^{(k)}
\end{gathered}
$$

be the total enhanced parameters values;

iii) evaluate the internal force vectors $\left.\boldsymbol{f}_{\text {int }}\left(\overline{\boldsymbol{u}}^{h},[|\boldsymbol{\varepsilon}|], \overline{\boldsymbol{p}}_{\boldsymbol{f}}^{h}, \tilde{p}_{f}^{h}\right)\right|_{n+1} ^{(k)}$ and $\boldsymbol{f}_{\mathbf{1 i n \boldsymbol { t }}}\left(\Delta \overline{\boldsymbol{u}}^{h}, \Delta[|\boldsymbol{\varepsilon}|], \overline{\boldsymbol{p}}_{\boldsymbol{f}}{ }^{h}, \Delta \overline{\boldsymbol{p}}_{\boldsymbol{f}}{ }^{h}, \tilde{p}_{f}^{h}\right.$,

iv) check convergence: if it is satisfied then $\left.(\bullet)\right|_{n+1} ^{(k)}$ is the solution; otherwise, continue; and;

v) determine $\left.\Delta \overline{\boldsymbol{u}}^{h}\right|_{n+1} ^{(k)}$ and $\left.\Delta \overline{\boldsymbol{p}}_{\boldsymbol{f}}\right|_{n+1} ^{(k)}$, set $k \leftarrow k+1$, and go to step (i).

The only step in the solution procedure outlined above which remains to be addressed is determining $\left.\Delta \overline{\boldsymbol{u}}^{h}\right|_{n+1} ^{(k)}$ and $\left.\Delta \overline{\boldsymbol{p}}_{\boldsymbol{f}}\right|_{n+1} ^{(k)}$ (step (v)). This is detailed here after where, for sake of clarity, superscript and subscript are omitted.

Once the local system is solved thanks to algorithm (11), one has

$$
\begin{aligned}
& \boldsymbol{K}_{\boldsymbol{u} \mathrm{w}}{ }^{T} \Delta \overline{\boldsymbol{u}}^{h}+\boldsymbol{K}_{\mathrm{ww}} \Delta[|\varepsilon|]-\boldsymbol{K}_{\mathrm{w} \overline{\boldsymbol{p}}} \Delta \overline{\boldsymbol{p}}_{\boldsymbol{f}}^{h}-\boldsymbol{K}_{\mathrm{w} \tilde{p}} \Delta \tilde{p}_{f}^{h}=\mathbf{0}, \\
& \frac{1}{\Delta t} \boldsymbol{K}_{\boldsymbol{u} \tilde{p}}^{T} \Delta \overline{\boldsymbol{u}}^{h}+\frac{1}{\Delta t} \boldsymbol{K}_{\mathrm{w} \tilde{p}}^{T} \Delta[|\varepsilon|]+\left[\frac{1}{\Delta t} \mathcal{L}^{T}+\mathcal{G}^{T}\right] \Delta \overline{\boldsymbol{p}}_{\boldsymbol{f}}{ }^{h}+\left[\frac{1}{\Delta t} \mathcal{C}+\mathcal{B}\right] \Delta \tilde{p}_{f}^{h}=0,
\end{aligned}
$$

where it is now possible to express $\Delta[|\boldsymbol{\varepsilon}|]$ and $\Delta \tilde{p}_{f}^{h}$ as a function of $\Delta \overline{\boldsymbol{u}}^{h}$ and $\Delta \overline{\boldsymbol{p}}_{\boldsymbol{f}}{ }^{h}$ in order to perform a static condensation at the global level.

This gives the following expressions

$$
\begin{aligned}
\Delta[|\varepsilon|] & =-\left[\boldsymbol{K}_{\mathrm{ww}}+\frac{1}{\Delta t\left[\frac{1}{\Delta t} \mathcal{C}+\mathcal{B}\right]} \boldsymbol{K}_{\mathrm{w} \tilde{p}} \boldsymbol{K}_{\mathrm{w} \tilde{p}}^{T}\right]^{-1}\left[\left(\boldsymbol{K}_{\boldsymbol{u w}}{ }^{T}+\frac{1}{\Delta t\left[\frac{1}{\Delta t} \mathcal{C}+\mathcal{B}\right]} \boldsymbol{K}_{\mathrm{w} \tilde{p}} \boldsymbol{K}_{\boldsymbol{u} \tilde{p}}^{T}\right) \Delta \overline{\boldsymbol{u}}^{h}\right. \\
& \left.+\left(-\boldsymbol{K}_{\mathrm{w} \overline{\boldsymbol{p}}}+\frac{1}{\left[\frac{1}{\Delta t} \mathcal{C}+\mathcal{B}\right]} \boldsymbol{K}_{\mathrm{w} \tilde{p}}\left[\frac{1}{\Delta t} \mathcal{L}^{T}+\mathcal{G}^{T}\right]\right) \Delta \overline{\boldsymbol{p}}_{\boldsymbol{f}}{ }^{h}\right]
\end{aligned}
$$




$$
\begin{aligned}
\Delta \tilde{p}_{f}^{h} & =\left[-\frac{1}{\Delta t\left[\frac{1}{\Delta t} \mathcal{C}+\mathcal{B}\right]} \boldsymbol{K}_{\boldsymbol{u} \tilde{p}}^{T}+\frac{1}{\Delta t\left[\frac{1}{\Delta t} \mathcal{C}+\mathcal{B}\right]} \boldsymbol{K}_{\mathrm{w} \tilde{p}}^{T}\left(\boldsymbol{K}_{\mathrm{ww}}+\frac{1}{\Delta t\left[\frac{1}{\Delta t} \mathcal{C}+\mathcal{B}\right]} \boldsymbol{K}_{\mathrm{w} \tilde{p}} \boldsymbol{K}_{\mathrm{w} \tilde{p}}^{T}\right)^{-1}\right. \\
& \left.\left(\boldsymbol{K}_{\boldsymbol{u w}}{ }^{T}+\frac{1}{\Delta t\left[\frac{1}{\Delta t} \mathcal{C}+\mathcal{B}\right]} \boldsymbol{K}_{\mathrm{w} \tilde{p}} \boldsymbol{K}_{\boldsymbol{u} \tilde{p}}^{T}\right)\right] \Delta \overline{\boldsymbol{u}}^{h}+\left[-\frac{1}{\left[\frac{1}{\Delta t} \mathcal{C}+\mathcal{B}\right]}\left[\frac{1}{\Delta t} \mathcal{L}^{T}+\mathcal{G}^{T}\right]\right. \\
& +\frac{1}{\Delta t\left[\frac{1}{\Delta t} \mathcal{C}+\mathcal{B}\right]} \boldsymbol{K}_{\mathrm{w} \tilde{p}}^{T}\left(\boldsymbol{K}_{\mathrm{ww}}+\frac{1}{\Delta t\left[\frac{1}{\Delta t} \mathcal{C}+\mathcal{B}\right]} \boldsymbol{K}_{\mathrm{w} \tilde{p}} \boldsymbol{K}_{\mathrm{w} \tilde{p}}^{T}\right)^{-1}\left(-\boldsymbol{K}_{\mathrm{w} \bar{p}}+\right. \\
& \left.\left.\frac{1}{\left[\frac{1}{\Delta t} \mathcal{C}+\mathcal{B}\right]} \boldsymbol{K}_{\mathrm{w} \tilde{p}}\left[\frac{1}{\Delta t} \mathcal{L}^{T}+\mathcal{G}^{T}\right]\right)\right] \Delta \overline{\boldsymbol{p}}_{\boldsymbol{f}}{ }^{h}
\end{aligned}
$$

For sake of clarity, we set

$$
\begin{gathered}
\mathcal{X}=-\left[\boldsymbol{K}_{\mathrm{ww}}+\frac{1}{\Delta t\left[\frac{1}{\Delta t} \mathcal{C}+\mathcal{B}\right]} \boldsymbol{K}_{\mathrm{w} \tilde{p}} \boldsymbol{K}_{\mathrm{w} \tilde{p}}^{T}\right]^{-1}\left[\left(\boldsymbol{K}_{\boldsymbol{u w}}{ }^{T}+\frac{1}{\Delta t\left[\frac{1}{\Delta t} \mathcal{C}+\mathcal{B}\right]} \boldsymbol{K}_{\mathrm{w} \tilde{p}} \boldsymbol{K}_{\boldsymbol{u} \tilde{p}}^{T}\right)\right] \\
\mathcal{Y}=-\left[\boldsymbol{K}_{\mathrm{ww}}+\frac{1}{\Delta t\left[\frac{1}{\Delta t} \mathcal{C}+\mathcal{B}\right]} \boldsymbol{K}_{\mathrm{w} \tilde{p}} \boldsymbol{K}_{\mathrm{w} \tilde{p}}^{T}\right]^{-1}\left[\left(-\boldsymbol{K}_{\mathrm{w} \overline{\boldsymbol{p}}}+\frac{1}{\left[\frac{1}{\Delta t} \mathcal{C}+\mathcal{B}\right]} \boldsymbol{K}_{\mathrm{w} \tilde{p}}\left[\frac{1}{\Delta t} \mathcal{L}^{T}+\mathcal{G}^{T}\right]\right] .\right.
\end{gathered}
$$

and

$$
\begin{gathered}
\mathcal{W}=\left[-\frac{1}{\Delta t\left[\frac{1}{\Delta t} \mathcal{C}+\mathcal{B}\right]} \boldsymbol{K}_{\boldsymbol{u} \tilde{p}}^{T}+\frac{1}{\Delta t\left[\frac{1}{\Delta t} \mathcal{C}+\mathcal{B}\right]} \boldsymbol{K}_{\mathrm{w} \tilde{p}}^{T}\left(\boldsymbol{K}_{\mathrm{ww}}+\frac{1}{\Delta t\left[\frac{1}{\Delta t} \mathcal{C}+\mathcal{B}\right]} \boldsymbol{K}_{\mathrm{w} \tilde{p}} \boldsymbol{K}_{\mathrm{w} \tilde{p}}^{T}\right)^{-1}\right. \\
\left.\left(\boldsymbol{K}_{\boldsymbol{u} \mathrm{w}}{ }^{T}+\frac{1}{\Delta t\left[\frac{1}{\Delta t} \mathcal{C}+\mathcal{B}\right]} \boldsymbol{K}_{\mathrm{w} \tilde{p}} \boldsymbol{K}_{\boldsymbol{u} \tilde{p}}^{T}\right)\right] \\
\mathcal{Z}=\left[-\frac{1}{\left[\frac{1}{\Delta t} \mathcal{C}+\mathcal{B}\right]}\left[\frac{1}{\Delta t} \mathcal{L}^{T}+\mathcal{G}^{T}\right]+\frac{1}{\Delta t\left[\frac{1}{\Delta t} \mathcal{C}+\mathcal{B}\right]} \boldsymbol{K}_{\mathrm{w} \tilde{p}}^{T}\left(\boldsymbol{K}_{\mathrm{ww}}+\frac{1}{\Delta t\left[\frac{1}{\Delta t} \mathcal{C}+\mathcal{B}\right]} \boldsymbol{K}_{\mathrm{w} \tilde{p}} \boldsymbol{K}_{\mathrm{w} \tilde{p}}^{T}\right)^{-1}\right. \\
\left.\left(-\boldsymbol{K}_{\mathrm{w} \bar{p}}+\frac{1}{\left[\frac{1}{\Delta t} \mathcal{C}+\mathcal{B}\right]} \boldsymbol{K}_{\mathrm{w} \tilde{p}}\left[\frac{1}{\Delta t} \mathcal{L}^{T}+\mathcal{G}^{T}\right]\right)\right] .
\end{gathered}
$$

This gives

$$
\begin{aligned}
\Delta[|\varepsilon|] & =\mathcal{X} \Delta \overline{\boldsymbol{u}}^{h}+\mathcal{Y} \Delta \overline{\boldsymbol{p}}_{\boldsymbol{f}}{ }^{h}, \\
\Delta \tilde{p}_{f}^{h} & =\mathcal{W} \Delta \overline{\boldsymbol{u}}^{h}+\mathcal{Z} \Delta \overline{\boldsymbol{p}}_{\boldsymbol{f}}{ }^{h} .
\end{aligned}
$$

\subsection{Static condensation}

We now come back to the global equation (68) and (172) to perform a static condensation ([34]). In (72), we express $\Delta \overline{\boldsymbol{p}}_{\boldsymbol{f}}{ }^{h}$ as a function of $\Delta \overline{\boldsymbol{u}}^{h}$. 
This gives, using (100) and (101)

$$
\begin{aligned}
& {\left[\frac{1}{\Delta t} \boldsymbol{K}_{\boldsymbol{u} \overline{\boldsymbol{p}}}^{T}+\frac{1}{\Delta t} \boldsymbol{K}_{\mathrm{w} \overline{\boldsymbol{p}}}^{T} \mathcal{X}+\left[\frac{1}{\Delta t} \mathcal{L}+\mathcal{G}\right] \mathcal{W}\right] \Delta \overline{\boldsymbol{u}}^{h}+\left[\left[\frac{1}{\Delta t} \mathcal{M}+\mathcal{H}\right]\right.} \\
& \left.+\frac{1}{\Delta t} \boldsymbol{K}_{\mathrm{w} \overline{\boldsymbol{p}}}^{T} \mathcal{Y}+\left[\frac{1}{\Delta t} \mathcal{L}+\mathcal{G}\right] \mathcal{Z}\right] \Delta \overline{\boldsymbol{p}}_{\boldsymbol{f}}{ }^{h}=-\left[\boldsymbol{f}_{\mathbf{1}_{\text {int }}}-\boldsymbol{f}_{\mathbf{1}_{\text {ext }}}\right]
\end{aligned}
$$

Finally $\Delta \overline{\boldsymbol{p}}_{\boldsymbol{f}}{ }^{h}$ can be rewritten in terms of $\Delta \overline{\boldsymbol{u}}^{h}$ only such as

$$
\begin{aligned}
\Delta \overline{\boldsymbol{p}}_{\boldsymbol{f}}{ }^{h} & =-\left[\left[\frac{1}{\Delta t} \mathcal{M}+\mathcal{H}\right]+\frac{1}{\Delta t} \boldsymbol{K}_{\mathrm{w} \overline{\boldsymbol{p}}}^{T} \mathcal{Y}+\left[\frac{1}{\Delta t} \mathcal{L}+\mathcal{G}\right] \mathcal{Z}\right]^{-1}\left[\left[\boldsymbol{f}_{\boldsymbol{1}_{\text {int }}}-\boldsymbol{f}_{\boldsymbol{1}_{\text {ext }}}\right]\right. \\
& \left.+\left[\frac{1}{\Delta t} \boldsymbol{K}_{\boldsymbol{u} \overline{\boldsymbol{p}}}^{T}+\frac{1}{\Delta t} \boldsymbol{K}_{\mathrm{w} \overline{\boldsymbol{p}}}^{T} \mathcal{X}+\left[\frac{1}{\Delta t} \mathcal{L}+\mathcal{G}\right] \mathcal{W}\right] \Delta \overline{\boldsymbol{u}}^{h}\right]
\end{aligned}
$$

For sake of clarity, we set

$$
\mathcal{U}=\left[\left[\frac{1}{\Delta t} \mathcal{M}+\mathcal{H}\right]+\frac{1}{\Delta t} \boldsymbol{K}_{\mathrm{w} \bar{p}}^{T} \mathcal{Y}+\left[\frac{1}{\Delta t} \mathcal{L}+\mathcal{G}\right] \mathcal{Z}\right]
$$

and

$$
\mathcal{V}=\left[\frac{1}{\Delta t} \boldsymbol{K}_{u \bar{p}}^{T}+\frac{1}{\Delta t} \boldsymbol{K}_{\mathrm{w} \bar{p}}^{T} \mathcal{X}+\left[\frac{1}{\Delta t} \mathcal{L}+\mathcal{G}\right] \mathcal{W}\right]
$$

This gives

$$
\Delta \overline{\boldsymbol{p}}_{\boldsymbol{f}}{ }^{h}=-\mathcal{U}^{-1}\left[\left[\boldsymbol{f}_{\mathbf{1}_{\text {int }}}-\boldsymbol{f}_{\boldsymbol{1}_{\text {ext }}}\right]+\mathcal{V} \Delta \overline{\boldsymbol{u}}^{h}\right] .
$$

Inserting equations (100), (101) and (106) in (68) gives

$$
\begin{aligned}
& \boldsymbol{K}_{\boldsymbol{u u}} \Delta \overline{\boldsymbol{u}}^{h}+\boldsymbol{K}_{\boldsymbol{u w}}\left[\boldsymbol{\mathcal { X }} \Delta \overline{\boldsymbol{u}}^{h}-\mathcal{Y} \mathcal{U}^{-1}\left[\left(\boldsymbol{f}_{\mathbf{1 i n t}}-\boldsymbol{f}_{\mathbf{1}_{\text {ext }}}\right)+\mathcal{V} \Delta \overline{\boldsymbol{u}}^{h}\right]\right] \\
& +\boldsymbol{K}_{\boldsymbol{u} \bar{p}} \mathcal{U}^{-1}\left[\left(\boldsymbol{f}_{\mathbf{1}_{\text {int }}}-\boldsymbol{f}_{\mathbf{1}_{\text {ext }}}\right)+\mathcal{V} \Delta \overline{\boldsymbol{u}}^{h}\right]-\boldsymbol{K}_{\boldsymbol{u} \tilde{p}}\left[\mathcal{W} \Delta \overline{\boldsymbol{u}}^{h}\right. \\
& \left.-\mathcal{Z U}^{-1}\left[\left(f_{1_{\text {int }}}-\boldsymbol{f}_{1_{\text {ext }}}\right)+\mathcal{V} \Delta \overline{\boldsymbol{u}}^{h}\right]\right]=-\left[\boldsymbol{f}_{\text {int }}-\boldsymbol{f}_{\text {ext }}\right] \text {. }
\end{aligned}
$$

After collecting terms together and reordering the former expression, one gets

$$
\begin{aligned}
& {\left[\boldsymbol{K}_{\boldsymbol{u} \boldsymbol{u}}+\boldsymbol{K}_{\boldsymbol{u} \mathrm{w}}\left(\mathcal{X}-\mathcal{Y} \mathcal{U}^{-1} \mathcal{V}\right)+\boldsymbol{K}_{\boldsymbol{u} \bar{p}} \mathcal{U}^{-1} \mathcal{V}-\boldsymbol{K}_{\boldsymbol{u} \tilde{p}}\left(\mathcal{W}-\mathcal{Z} \mathcal{U}^{-1} \mathcal{V}\right)\right] \Delta \overline{\boldsymbol{u}}^{h}} \\
& =-\left[\boldsymbol{f}_{\boldsymbol{i n t}}-\boldsymbol{f}_{\text {ext }}\right]-\left[-\boldsymbol{K}_{\boldsymbol{u} \mathrm{w}} \mathcal{Y} \mathcal{U}^{-1}+\boldsymbol{K}_{\boldsymbol{u} \bar{p}} \mathcal{U}^{-1}+\boldsymbol{K}_{\boldsymbol{u} \tilde{p}} \mathcal{Z U}^{-1}\right]\left[\boldsymbol{f}_{\boldsymbol{1}_{\text {int }}}-\boldsymbol{f}_{\text {1ext }_{\text {ex }}}\right]
\end{aligned}
$$

This can be written under the form

$$
\hat{\boldsymbol{K}} \Delta \overline{\boldsymbol{u}}^{h}=\hat{\boldsymbol{f}} .
$$


where

$$
\hat{\boldsymbol{K}}=\left[\boldsymbol{K}_{\boldsymbol{u u}}+\boldsymbol{K}_{\boldsymbol{u} \mathrm{w}}\left(\mathcal{X}-\mathcal{Y} \mathcal{U}^{-1} \mathcal{V}\right)+\boldsymbol{K}_{\boldsymbol{u} \bar{p}} \mathcal{U}^{-1} \mathcal{V}-\boldsymbol{K}_{\boldsymbol{u} \tilde{p}}\left(\mathcal{W}-\mathcal{Z} \mathcal{U}^{-1} \mathcal{V}\right)\right]
$$

and

$$
\hat{\boldsymbol{f}}=-\left[\boldsymbol{f}_{\text {int }}-\boldsymbol{f}_{\text {ext }}\right]-\left[-\boldsymbol{K}_{\boldsymbol{u w}} \mathcal{Y} \mathcal{U}^{-1}+\boldsymbol{K}_{\boldsymbol{u} \bar{p}} \mathcal{U}^{-1}+\boldsymbol{K}_{\boldsymbol{u} \tilde{p}} \mathcal{Z} \mathcal{U}^{-1}\right]\left[\boldsymbol{f}_{1_{\text {int }}}-\boldsymbol{f}_{\mathbf{1}_{\text {ext }}}\right]
$$

Once the value of $\Delta \overline{\boldsymbol{u}}^{h}$ is computed, the value of $\Delta \overline{\boldsymbol{p}}_{f}{ }^{h}$ is post-calculated using (106).

\section{Conclusion}

We have presented in this paper a method to model a poro-elastic fully saturated twophase material at the mesoscopic scale. In particular, a $\mathrm{W} / \mathrm{W}$ enhancement (discontinuous strain and pressure gradient fields) implementation has been presented in the context of the Embedded Finite Element method with the corresponding discretization of the strain and pressure gradient enhanced fields, giving a contribution to the enhancements presented in Table 1. In parallel, an efficient local-global algorithmic treatment is proposed: the enhanced fields are introduced at the element level as a local enhancement of the strain and pressure gradient fields and this local structure allows the static condensation of the enhanced parameters, leading altogether to an efficient solution of these problems.

Some extensions of the method presented in this paper are also regarded, starting with the extension to partially-saturated two-phase media. This can be relevant in the context of moisture transport problem ([36]) or diffusion problem ([37]) in heterogeneous media. Furthermore, consideration of crack initiation and propagation in the mortar matrix and debonding at the interface between aggregates and mortar matrix is also of our main interest. On the one hand, for the mechanical problem, this can be achieved using a strong enhancement by the means of the Strong Discontinuity Approach (SDA), introducing an Heaviside function in the displacement field. This method is well-known and massively used in the literature (see [10], [38] or [29] for instance). On the other hand regarding the literature, for the fluid problem, this enhancement can be achieved in different ways. Thus some authors consider (1) a continuous pressure field (see [15], [39], [20]) then introducing 
a weak discontinuity in the gradient of the pressure field as performed in this paper and (2) a discontinuous pressure field (see [40], [21]) by introducing a strong discontinuity in the pressure field. By keeping the $\mathrm{W} / \mathrm{W}$ enhancement already done in this work for capturing heterogeneities, two new enhancements can be achieved: (1) a $\mathrm{S}+\mathrm{W} / \mathrm{W}$ if the pressure is continuous and (2) a $\mathrm{S}+\mathrm{W} / \mathrm{S}+\mathrm{W}$ if the pressure is discontinuous. Finally, introducing the coupling of fluid transport with the process of cracking is of main interest in Civil Engineering problems such as $\mathrm{CO}_{2}$ storage or nuclear industry.

\section{Appendix A. details for $\tilde{\varepsilon}$}

In order to obtain $\tilde{\boldsymbol{\varepsilon}}$, we apply the symmetric gradient operator to $\tilde{\boldsymbol{u}}$, this yields

$$
\tilde{\boldsymbol{\varepsilon}}=\boldsymbol{\nabla}^{s}(\tilde{\boldsymbol{u}})=\left(\begin{array}{ccc}
\tilde{u}_{1,1} & \frac{1}{2}\left(\tilde{u}_{1,2}+\tilde{u}_{2,1}\right) & \frac{1}{2}\left(\tilde{u}_{1,3}+\tilde{u}_{3,1}\right) \\
\operatorname{sym} & \tilde{u}_{2,2} & \frac{1}{2}\left(\tilde{u}_{2,3}+\tilde{u}_{3,2}\right) \\
\operatorname{sym} & \operatorname{sym} & \tilde{u}_{3,3}
\end{array}\right),
$$

where $\tilde{\boldsymbol{u}}=\left(\tilde{u}_{1}, \tilde{u}_{2}, \tilde{u}_{3}\right), \boldsymbol{x}=\left(x_{1}, x_{2}, x_{3}\right)$ and $(\bullet, 1),\left(\bullet_{, 2}\right)$ and $\left(\bullet_{, 3}\right)$ represent derivatives in relation with $x_{1}, x_{2}$ and $x_{3}$.

Following the reasoning developed in $([32])$, we have for $\tilde{u}_{1,1}$

$$
\tilde{u}_{1,1}=\frac{\partial \tilde{u}_{1}}{\partial x_{1}}=\frac{\partial \tilde{u}_{1}}{\partial\left(n_{k} \cdot(x-\xi)_{k}\right)} \frac{\partial\left(n_{k} \cdot(x-\xi)_{k}\right)}{\partial x_{1}}=\tilde{u}_{1}^{\prime} n_{1}=\Theta\left([\varepsilon]_{n} n_{1}+[\varepsilon]_{m} m_{1}+[\varepsilon]_{t} t_{1}\right) n_{1}
$$

where $n_{k} \cdot(x-\xi)_{k}=n_{1} \cdot(x-\xi)_{1}+n_{2} \cdot(x-\xi)_{2}+n_{3} \cdot(x-\xi)_{3}$.

And so

$$
\begin{aligned}
& \frac{\partial \tilde{u}_{1}}{\partial x_{2}}=\Theta\left([\varepsilon]_{n} n_{1}+[\varepsilon]_{m} m_{1}+[\varepsilon]_{t} t_{1}\right) n_{2}, \\
& \frac{\partial \tilde{u}_{2}}{\partial x_{1}}=\Theta\left([\varepsilon]_{n} n_{2}+[\varepsilon]_{m} m_{2}+[\varepsilon]_{t} t_{2}\right) n_{1}, \\
& \frac{\partial \tilde{u}_{2}}{\partial x_{2}}=\Theta\left([\varepsilon]_{n} n_{2}+[\varepsilon]_{m} m_{2}+[\varepsilon]_{t} t_{2}\right) n_{2},
\end{aligned}
$$




$$
\begin{aligned}
& \frac{\partial \tilde{u}_{3}}{\partial x_{3}}=\Theta\left([\varepsilon]_{n} n_{3}+[\varepsilon]_{m} m_{3}+[\varepsilon]_{t} t_{3}\right) n_{3}, \\
& \frac{\partial \tilde{u}_{1}}{\partial x_{3}}=\Theta\left([\varepsilon]_{n} n_{1}+[\varepsilon]_{m} m_{1}+[\varepsilon]_{t} t_{1}\right) n_{3}, \\
& \frac{\partial \tilde{u}_{3}}{\partial x_{1}}=\Theta\left([\varepsilon]_{n} n_{3}+[\varepsilon]_{m} m_{3}+[\varepsilon]_{t} t_{3}\right) n_{1}, \\
& \frac{\partial \tilde{u}_{2}}{\partial x_{3}}=\Theta\left([\varepsilon]_{n} n_{2}+[\varepsilon]_{m} m_{2}+[\varepsilon]_{t} t_{2}\right) n_{3}, \\
& \frac{\partial \tilde{u}_{3}}{\partial x_{2}}=\Theta\left([\varepsilon]_{n} n_{3}+[\varepsilon]_{m} m_{3}+[\varepsilon]_{t} t_{3}\right) n_{2} .
\end{aligned}
$$

Developing the former expressions gives

$$
\begin{gathered}
\tilde{\epsilon}_{11}=\Theta\left([\varepsilon]_{n} n_{1}^{2}+[\varepsilon]_{m} m_{1} n_{1}+[\varepsilon]_{t} t_{1} n_{1}\right), \\
\tilde{\epsilon}_{22}=\Theta\left([\varepsilon]_{n} n_{2}^{2}+[\varepsilon]_{m} m_{2} n_{2}+[\varepsilon]_{t} t_{2} n_{2}\right), \\
\tilde{\epsilon}_{33}=\Theta\left([\varepsilon]_{n} n_{3}^{2}+[\varepsilon]_{m} m_{3} n_{3}+[\varepsilon]_{t} t_{3} n_{3}\right), \\
\tilde{\epsilon}_{12}=\frac{1}{2} \Theta\left([\varepsilon]_{n}\left(n_{1} n_{2}+n_{2} n_{1}\right)+[\varepsilon]_{m}\left(m_{1} n_{2}+m_{2} n_{1}\right)+[\varepsilon]_{t}\left(t_{1} n_{2}+t_{2} n_{1}\right)\right), \\
\tilde{\epsilon}_{13}=\frac{1}{2} \Theta\left([\varepsilon]_{n}\left(n_{1} n_{3}+n_{3} n_{1}\right)+[\varepsilon]_{m}\left(m_{1} n_{3}+m_{3} n_{1}\right)+[\varepsilon]_{t}\left(t_{1} n_{3}+t_{3} n_{1}\right)\right), \\
\tilde{\epsilon}_{23}=\frac{1}{2} \Theta\left([\varepsilon]_{n}\left(n_{2} n_{3}+n_{3} n_{2}\right)+[\varepsilon]_{m}\left(m_{2} n_{3}+m_{3} n_{2}\right)+[\varepsilon]_{t}\left(t_{2} n_{3}+t_{3} n_{2}\right)\right) .
\end{gathered}
$$

Collecting terms together yields

$$
\tilde{\varepsilon}=\Theta[\varepsilon]_{n}\left(\begin{array}{ccc}
n_{1}^{2} & n_{1} n_{2} & n_{1} n_{3} \\
n_{1} n_{2} & n_{2}^{2} & n_{2} n_{3} \\
n_{1} n_{3} & n_{2} n_{3} & n_{3}^{2}
\end{array}\right)+
$$




$$
\begin{gathered}
\Theta[\varepsilon]_{m}\left(\begin{array}{ccc}
m_{1} n_{1} & \frac{1}{2}\left(m_{1} n_{2}+m_{2} n_{1}\right) & \frac{1}{2}\left(m_{1} n_{3}+m_{3} n_{1}\right) \\
\frac{1}{2}\left(m_{1} n_{2}+m_{2} n_{1}\right) & m_{2} n_{2} & \frac{1}{2}\left(m_{2} n_{3}+m_{3} n_{2}\right) \\
\frac{1}{2}\left(m_{1} n_{3}+m_{3} n_{1}\right) & \frac{1}{2}\left(m_{2} n_{3}+m_{3} n_{2}\right) & m_{3} n_{3}
\end{array}\right)+ \\
\Theta[\varepsilon]_{t}\left(\begin{array}{ccc}
t_{1} n_{1} & \frac{1}{2}\left(t_{1} n_{2}+t_{2} n_{1}\right) & \frac{1}{2}\left(t_{1} n_{3}+t_{3} n_{1}\right) \\
\frac{1}{2}\left(t_{1} n_{2}+t_{2} n_{1}\right) & t_{2} n_{2} & \frac{1}{2}\left(t_{2} n_{3}+t_{3} n_{2}\right) \\
\frac{1}{2}\left(t_{1} n_{3}+t_{3} n_{1}\right) & \frac{1}{2}\left(t_{2} n_{3}+t_{3} n_{2}\right) & t_{3} n_{3}
\end{array}\right) .
\end{gathered}
$$

Finally $\tilde{\varepsilon}$ can be rewritten under the form

$$
\tilde{\boldsymbol{\varepsilon}}=\Theta\left([\varepsilon]_{n} \boldsymbol{n} \otimes \boldsymbol{n}+\frac{[\varepsilon]_{m}}{2}(\boldsymbol{n} \otimes \boldsymbol{m})^{s}+\frac{[\varepsilon]_{t}}{2}(\boldsymbol{n} \otimes \boldsymbol{t})^{s}\right) .
$$

\section{References}

[1] T. Strouboulis, I. Babuška, K. Copps, The design and analysis of the Generalized Finite Element Method, Computer Methods in Applied Mechanics and Enginnering 181 (2000) 43-69.

[2] T. Strouboulis, K. Copps, I. Babuška, The generalized finite element method: an example of its implementation and illustration of its performance, International Journal for Numerical Methods in Engineering 47 (2000) 1401-1417.

[3] T. Belytschko, N. Moës, S. Usui, C. Parimi, Arbitrary discontinuities in finite elements, International Journal for Numerical Methods in Engineering 50 (4) (2001) 993-1013.

[4] N. Moës, J. Dolbow, T. Belytschko, A finite element method for crack growth without remeshing, International Journal of Numerical Methods in Engineering 46 (1999) 131-150.

[5] S. Mariani, U. Perego, Extended finite element method for quasi-brittle fracture, International Journal for Numerical Methods in Engineering 58 (1) (2003) 103-126.

[6] F. Armero, K. Garikipati, An analysis of strong discontinuities in multiplicative finite strain plasticity and their relation with the numerical simulation of strain localization in solids, International Journal of Solids and Structures 33 (20-22) (1996) 2863-2885.

[7] J. Oliver, Modeling strong discontinuities in solid mechanics via strain softening constitutive equations. Part 2: numerical simulation, International Journal for Numerical Methods in Engineering 39 (21) (1996) 3601-3623.

[8] R. I. Borja, A finite element model for strain localization analysis of strongly discontinuous fields based on standard galerkin approximation, Computer Methods in Applied Mechanics and Engineering $190(11-12)(2000)$ 1529-1549. 
[9] N. Benkemoun, M. Hautefeuille, J.-B. Colliat, A. Ibrahimbegovic, Modeling heterogeneous materials failure: 3D meso-scale models with embedded discontinuities, International Journal of Numerical Methods in Engineering 82 (2010) 1671-1688.

[10] J. C. Simo, J. Oliver, F. Armero, An analysis of strong discontinuities induced by strain-softening in rate independent inelastic solids, Computational Mechanics 12 (1993) 277-296.

[11] M. Ortiz, Y. Leroy, A. Needleman, A finite element method for localized failure analysis, Computer Methods in Applied Mechanics and Enginnering 61 (1987) 189-214.

[12] O. Coussy, Poromechanics, Wiley, 2004.

[13] K. Wilmanski, A thermodynamic model of compressible porous materials with the balance equation of porosity, Transport in Porous Media 32 (1) (1998) 21-47.

[14] B. Schrefler, R. Lewis, The finite element method in the static and dynamic deformation and consolidation of porous media, John Wiley, 1998.

[15] J. Alfaiate, P. Moonen, L. Sluys, J. Carmeliet, On the use of strong discontinuity formulations for the modeling of preferential moisture uptake in fractured porous media, Computer Methods in Applied Mechanics and Engineering 199 (45-48) (2010) 2828-2839.

[16] X. Jourdain, J.-B. Colliat, C. DeSa, F. Benboudjema, F. Gatuingt, Upscaling permeability for fractured concrete: meso-macro numerical approach coupled to strong discontinuities, International Journal for Numerical and Analytical Methods in Geomechanics 38 (2013) 536-550.

[17] V.-M. Ngo, A. Ibrahimbegovic, D. Brancherie, Model for localized failure with thermo-plastic coupling: Theoretical formulation and ED-FEM implementation, Computers \& Structures 127 (0) (2013) 2-18.

[18] C. Callari, F. Armero, Finite element methods for the analysis of strong discontinuities in coupled poro-plastic media, Computer Methods in Applied Mechanics and Engineering 191 (39-40) (2002) 4371-4400.

[19] C. Callari, F. Armero, A. Abati, Strong discontinuities in partially saturated poroplastic solids, Computer Methods in Applied Mechanics and Engineering 199 (23-24) (2010) 1513-1535.

[20] J. Réthoré, R. de Borst, M.-A. Abellan, A two-scale approach for fluid flow in fractured porous media, International Journal for Numerical Methods in Engineering 71 (7) (2007) 780-800.

[21] R. de Borst, J. C. Remmers, A. Needleman, M.-A. Abellan, Discrete vs smeared crack models for concrete fracture: bridging the gap, International Journal for Numerical and Analytical Methods in Geomechanics 28 (7-8) (2004) 583-607.

[22] T. Mohammadnejad, A. Khoei, An extended finite element method for hydraulic fracture propagation in deformable porous media with the cohesive crack model, Finite Elements in Analysis and Design 73 (0) (2013) 77-95.

[23] I. Maruyama, H. Sasano, Strain and crack distribution in concrete during drying, Materials and Struc- 
tures 47 (3) (2014) 517-532.

[24] I. Ö. Yaman, N. Hearn, H. M. Aktan, Active and non-active porosity in concrete. part I: experimental evidence., Materials and Structures 35 (2002) 102-109.

[25] N. Moës, M. Cloirec, P. Cartraud, J.-F. Remacle, A computational approach to handle complex microstructure geometries, Computer Methods in Applied Mechanics and Engineering 192 (2003) 31633177.

[26] K. Washizu, Variational methods in elasticity and plasticity, 3rd Edition, Pergamon Press, New York, 1982.

[27] J. C. Simo, M. Rifai, A class of mixed assumed strain methods and the method of incompatible modes., International Journal of Numerical Methods in Engineering 29 (1990) 1595-1638.

[28] J. Simo, F. Armero, R. Taylor, Improved versions of assumed enhanced strain tri-linear elements for 3D finite deformation problems, Computer Methods in Applied Mechanics and Engineering 110 (1993) $359-386$.

[29] E. Roubin, A. Vallade, N. Benkemoun, J.-B. Colliat, Multi-scale failure of heterogeneous materials: a double kinematics enhancement for EFEM method, International Journal of Solids and Structures 52 (2015) 180-196.

[30] C. Tamagnini, Computational Soil Dynamics, aLERT School : Soil - Structure Interaction. Aussois, Septembre 2013.

[31] D. Markovič, Modélisation multi-échelles de structures hétérogènes aux comportements anélastiques non-linéaires, Ph.D. thesis, École Normal Supérieure de Cachan (2004).

[32] R. de Borst, G.-N. Wells, L.-J. Sluys, Some observations on embedded discontinuity models, Engineering Computations 18 (2001) 241-254.

[33] M. A. Biot, General theory of three-dimensional consolidation, Journal of applied physics 12 (2) (2004) 155-164.

[34] E. L. Wilson, The static condensation algorithm, International Journal for Numerical Methods in Engineering 8 (1974) 198-203.

[35] J. C. Simo, T. J. R. Hughes, Computational Inelasticity, Interdisciplinary Applied Mathematics, Springer Verlag, New York, Berlin, Heidelberg, 1997.

[36] J. Carmeliet, S. Roels, Determination of the isothermal moisture transport properties of porous building materials, Journal of Building Physics 24 (3) (2001) 183-210.

[37] B. Šavija, M. Luković, E. Schlangen, Lattice modeling of rapid chloride migration in concrete, Cement and Concrete Research 6162 (0) (2014) 49 - 63.

[38] G. N. Wells, L. J. Sluys, Application of embedded discontinuities for softening solids, Engineering Fracture Mechanics 65 (2000) 263-281. 
[39] F. Armero, C. Callari, An analysis of strong discontinuities in a saturated poro-plastic solid, International Journal for Numerical Methods in Engineering 46 (10) (1999) 1673-1698.

[40] R. Larsson, K. Runesson, S. Sture, Embedded localization band in undrained soil based on regularized strong discontinuity-theory and FE-analysis, International Journal of Solids and Structures 33 (20-22) (1996) 3081-3101. 